\title{
ALGEBRAIC DEPENDENCE OF MEROMORPHIC MAPPINGS IN VALUE DISTRIBUTION THEORY
}

\author{
YOSHIHIRO AIHARA*
}

\begin{abstract}
In this paper we first prove some criteria for the propagation of algebraic dependence of dominant meromorphic mappings from an analytic finite covering space $X$ over the complex $m$-space into a projective algebraic manifold. We study this problem under a condition on the existence of meromorphic mappings separating the generic fibers of $X$. We next give applications of these criteria to the uniqueness problem of meromorphic mappings. We deduce unicity theorems for meromorphic mappings and also give some other applications. In particular, we study holomorphic mappings into a smooth elliptic curve $E$ and give conditions under which two holomorphic mappings from $X$ into $E$ are algebraically related.
\end{abstract}

\section{Introduction}

Let $f_{1}, \ldots, f_{l}$ be dominant meromorphic mappings from a finite analytic (ramified) covering space $\pi: X \rightarrow \mathbb{C}^{m}$ into a projective algebraic manifold $M$. Suppose that they have the same inverse images of given divisors on $M$. Our aim in this paper is to seek conditions under which $f_{1}, \ldots, f_{l}$ are algebraically related. We study this problem from the point of view in value distribution theory. Roughly speaking, our results say that if these mappings satisfy the same algebraic relation at all points of the set of the inverse images of divisors and if the given divisors are sufficiently ample, then they must satisfy this relationship identically. These results are considered as the propagation theorems of algebraic dependence. The propagation of dependence from a proper analytic subset to the whole space was first studied by L. Smiley [17] (cf. [19, p. 176]). There have been several studies on the propagation of dependence (cf. [5], [6], [8] and [21]). So far, this problem has been studied under the conditions on the growth of meromorphic mappings. However, there can be only a few restricted cases where meromorphic

Received September 13, 2000.

2000 Mathematics Subject Classification: Primary 32H30; Secondary 32H04.

* Research supported in part by the Grants-in-Aid for Scientific Research, The Ministry of Education, Science and Culture, Japan. 
mappings satisfying these conditions exist. In this paper we first give some criteria for the propagation of algebraic dependence of meromorphic mappings from $X$ into $M$ under the condition on the existence of meromorphic mappings separating the fibers of $\pi: X \rightarrow \mathbb{C}^{m}$. Thanks to the theory of algebroid reduction of meromorphic mappings, we can always find such a mapping. Thus it seems that our condition is more natural and essential than the above mentioned conditions. The theorem on algebroid reduction of meromorphic mappings and the ramification estimate due to J. Noguchi [11] are essentially important in the proofs of our results. In some of our criteria, we assume somewhat complicated conditions. However, they have a wide range of applicability and give sharpness. These criteria are actually corollaries of two basic theorems, which are Theorems 2.2 and 2.15 in Section 2. In the next part of this paper, we give their applications to the uniqueness problem of meromorphic mappings. For example, we study the uniqueness problem of holomorphic mappings into smooth elliptic curves.

Let $L$ be a line bundle over $M$. We denote by $H^{0}(M, L)$ the space of all holomorphic sections of $L \rightarrow M$. A line bundle $L$ over $M$ is said to be big provided that

$$
\operatorname{dim} H^{0}(M, \nu L) \geq C \nu^{\operatorname{dim} M}
$$

for all sufficiently large positive integers $\nu$ and for some positive constant $C$. Let $\operatorname{Pic}(M)$ be the Picard group over $M$. Let $F \in \operatorname{Pic}(M) \otimes \mathbb{Q}$ and $\gamma \in \mathbb{Q}$. We simply write $\gamma F$ for $F^{\otimes \gamma}$. Then $F$ is said to be big (resp. ample) provided that a line bundle $\nu F \in \operatorname{Pic}(M)$ is big (resp. ample) for some positive integer $\nu$. We fix an ample line bundle $L \rightarrow M$. Let $D_{1}, \ldots, D_{q}$ be divisors in $|L|$ such that $D_{1}+\cdots+D_{q}$ has only simple normal crossings, where $|L|$ is the complete linear system defined by $L$. Let $S_{1}, \ldots, S_{q}$ be hypersurfaces in $X$ such that $\operatorname{dim} S_{i} \cap S_{j} \leq m-2$ for any $i$ and $j(i \neq j)$. We define a hypersurface $S$ in $X$ by $S=S_{1} \cup \cdots \cup S_{q}$. Assume that, for each $i$, the union of all irreducible components of $f_{i}^{*} D_{j}$ with the multiplicities at most $k_{j}$ coincides with $S_{j}$ for all $j$ with $1 \leq j \leq q$, where $k_{j}$ is a fixed positive integer. Set $M^{l}=M \times \cdots \times M$ (l-times). Suppose that $f_{1}, \ldots, f_{l}$ are algebraically dependent on $S$, that is, $\left(f_{1} \times \cdots \times f_{l}\right)(S) \subseteq \Sigma$ for some indecomposable hypersurface $\Sigma$ in $M^{l}$. By Noguchi's theorem on the algebroid reduction of meromorphic mappings, we may assume that each $f_{j}: X \rightarrow M$ separates the fibers of $\pi: X \rightarrow \mathbb{C}^{m}$. Since $L$ is ample, there exist the least positive integer $\mu_{0}$ and a pair of sections $\sigma_{0}, \sigma_{1} \in$ $H^{0}\left(M, \mu_{0} L\right)$ such that each meromorphic function $f_{j}^{*}\left(\sigma_{0} / \sigma_{1}\right)$ separates the 
fibers of $\pi: X \rightarrow \mathbb{C}^{m}$. Let $F_{0}$ be a big line bundle over $M$. We denote by $s_{0}$ the sheet number of $\pi: X \rightarrow \mathbb{C}^{m}$. Set $k_{0}=\max _{1 \leq j \leq l} k_{j}$. We define $L_{0} \in \operatorname{Pic}(M) \otimes \mathbb{Q}$ by

$$
L_{0}=\left(\sum_{j=1}^{q} \frac{k_{j}}{k_{j}+1}-2 \mu_{0}\left(s_{0}-1\right)\right) L \otimes\left(-\frac{\tilde{\gamma} l k_{0}}{k_{0}+1} F_{0}\right),
$$

where $\tilde{\gamma}$ is a positive rational number depending only on $\Sigma$ and on $F_{0}$. Then we have our fundamental result, from which we see that the algebraic dependence on $S$ propagates to the whole space $X$ if $L_{0}$ is sufficiently big.

TheOREM. Suppose that $f_{1}, \ldots, f_{l}$ are algebraically dependent on $S$. If $L_{0} \otimes K_{M}$ is big, then $f_{1}, \ldots, f_{l}$ are algebraically dependent on $X$.

In Section 2, we give the proof of the above theorem, which is just Theorem 2.2. Furthermore, we consider the case where given divisors may determine distinct line bundles and Theorem 2.15 is fundamental in this case. We also study the effect of the existence of deficient divisors to the propagation of dependence. In Sections 3-5, we will give their applications. These sections are actually the principal part of this paper. We note that a certain kind of unicity theorems such as results in [2] and [4] may be considered as a special case of theorems on the propagation of dependence.

In these theorems we can see that, for two meromorphic mappings $f, g: X \rightarrow M$ with the same inverse images of divisors as point sets (say $Z$ ) satisfying $f=g$ on $Z$, the algebraic relation $f=g$ on $Z$ propagates to the whole space $X$. We prove some unicity theorems from this point of view in Section 3. In Section 4, we investigate meromorphic mappings into complex projective spaces and give theorems on algebraic dependence. We finally study the uniqueness problem of holomorphic mappings into smooth elliptic curves in Section 5. In particular, we give some conditions under which two holomorphic mappings are related by endomorphism of elliptic curves. The last section is devoted to giving the proof of Katsura's theorem, which is a crucial step in the proof of the main result in Section 5.

Acknowledgement. The author would like to thank Professor Toshiyuki Katsura for his useful advice. In particular, he has agreed with making his theorem public in this paper. 


\section{§1. Preliminaries}

Let $\pi: X \rightarrow \mathbb{C}^{m}$ be a finite analytic (ramified) covering space over $\mathbb{C}^{m}$ and let $s_{0}$ be its sheet number, that is, $X$ is a reduced irreducible normal complex space and $\pi: X \rightarrow \mathbb{C}^{m}$ is a proper surjective holomorphic mapping with discrete fibers. We denote by $B$ the ramification divisor. Let $z=\left(z_{1}, \ldots, z_{m}\right)$ be the natural coordinate system in $\mathbb{C}^{m}$, and set

$$
\|z\|^{2}=\sum_{\nu=1}^{m} z_{\nu} \bar{z}_{\nu}, \quad X(r)=\pi^{-1}\left(\left\{z \in \mathbb{C}^{m} ;\|z\|<r\right\}\right) \quad \text { and } \quad \alpha=\pi^{*} d d^{c}\|z\|^{2},
$$

where $d^{c}=(\sqrt{-1} / 4 \pi)(\bar{\partial}-\partial)$. For a $(1,1)$-current $\varphi$ of order zero on $X$ we set

$$
N(r, \varphi)=\frac{1}{s_{0}} \int_{1}^{r}\left\langle\varphi \wedge \alpha^{m-1}, \chi_{X(t)}\right\rangle \frac{d t}{t^{2 m-1}},
$$

where $\chi_{X(r)}$ denotes the characteristic function of $X(r)$. Let $M$ be a compact complex manifold and let $L \rightarrow M$ be a line bundle over $M$. Denote by $|\cdot|$ a hermitian fiber metric in $L$ and by $\omega$ its Chern form. Let $f: X \rightarrow M$ be a meromorphic mapping. We set

$$
T_{f}(r, L)=N\left(r, f^{*} \omega\right),
$$

and call it the characteristic function of $f$ with respect to $L$. We also define $T_{f}(r, F)$ for $F \in \operatorname{Pic}(M) \otimes \mathbb{Q}$ in the following way. If $\nu$ is a positive integer with $\nu F \in \operatorname{Pic}(M)$, then we set

$$
T_{f}(r, F)=\frac{1}{\nu} T_{f}(r, \nu F) .
$$

It is easy to see that $T_{f}(r, F)$ is well-defined. We have the following Nevanlinna's inequality for meromorphic mappings (cf. [11, p. 269]):

Theorem 1.1. Let $L \rightarrow M$ be a line bundle over $M$ and let $f: X \rightarrow$ $M$ be a nonconstant meromorphic mapping. Then

$$
N\left(r, f^{*} D\right) \leq T_{f}(r, L)+O(1)
$$

for $D \in|L|$ with $f(X) \nsubseteq \operatorname{Supp} D$, where $O(1)$ stands for a bounded term as $r \rightarrow+\infty$. 
Let $f: X \rightarrow M$ be a meromorphic mapping, and let $D \in|L|$. We define Nevanlinna's deficiency $\delta_{f}(D)$ by

$$
\delta_{f}(D)=1-\limsup _{r \rightarrow+\infty} \frac{N\left(r, f^{*} D\right)}{T_{f}(r, L)} .
$$

If $\delta_{f}(D)>0$, then $D$ is called a deficient divisor in the sense of Nevanlinna.

Let $Z$ be an effective divisor on $\mathbb{C}^{m}$ such that $Z=\sum_{j} \nu_{j} Z_{j}$ for distinct irreducible hypersurfaces $Z_{j}$ in $\mathbb{C}^{m}$ and for nonnegative integers $\nu_{j}$, and let $k$ be a positive integer. We set

$$
N_{k}(r, Z)=\sum_{j} \min \left\{k, \nu_{j}\right\} N\left(r, Z_{j}\right)
$$

A meromorphic mapping $f: X \rightarrow M$ is said to be dominant provided that $\operatorname{rank} f=\operatorname{dim} M$. The following second main theorem for dominant meromorphic mappings gives an essential computational way in the next section (cf. [11, Theorem 1]):

TheOREM 1.2. Let $M$ be a projective algebraic manifold with $m \geq$ $\operatorname{dim} M$ and let $L \rightarrow M$ be an ample line bundle. Suppose that $D_{1}, \ldots, D_{q}$ are divisors in $|L|$ such that $D_{1}+\cdots+D_{q}$ has only simple normal crossings. Let $f: X \rightarrow M$ be a dominant meromorphic mapping. Then

$$
q T_{f}(r, L)+T_{f}\left(r, K_{M}\right) \leq \sum_{j=1}^{q} N_{1}\left(r, f^{*} D_{j}\right)+N(r, B)+S_{f}(r),
$$

where $S_{f}(r)=O\left(\log T_{f}(r, L)\right)+o(\log r)$ except on a Borel subset $I \subseteq$ $[1,+\infty)$ with finite measure.

In applications of Theorem 1.2, it is essential to give the estimate for $N(r, B)$ by the characteristic function of $f$. In the case where $m=1$ and $M=\mathbb{P}_{1}(\mathbb{C})$, the ramification theorem due to H. Selberg is well-known (cf. [15]). In the case of meromorphic mappings $f: X \rightarrow M$, we have a following ramification estimate proved by J. Noguchi.

DeFinition 1.3. Let $Y$ be a compact complex manifold. We say that a meromorphic mapping $f: X \rightarrow Y$ separates the fibers of $\pi: X \rightarrow \mathbb{C}^{m}$, if there exists a point $z$ in $\mathbb{C}^{m}-\left(\operatorname{Supp} \pi_{*} B \cup \pi(I(f))\right)$ such that $f(x) \neq f(y)$ for any distinct points $x, y \in \pi^{-1}(z)$. In this case, $X$ is said to be the proper existence domain of $f$. 
Assume that $f: X \rightarrow M$ separates the fibers of $\pi: X \rightarrow M$. Since $L$ is ample, there exist the least positive integer $\mu_{0}$ and a pair of sections $\sigma_{0}, \sigma_{1} \in H^{0}\left(M, \mu_{0} L\right)$ such that a meromorphic function $f^{*}\left(\sigma_{0} / \sigma_{1}\right)$ separates the fibers of $\pi: X \rightarrow \mathbb{C}^{m}$. Then we have the following ramification estimate due to J. Noguchi ([11, p. 277]):

Theorem 1.4. (Noguchi) Suppose that $L \rightarrow M$ is ample and $f: X \rightarrow$ $M$ separates the fibers of $\pi: X \rightarrow M$. Let $\mu_{0}$ be as above. Then

$$
N(r, B) \leq 2 \mu_{0}\left(s_{0}-1\right) T_{f}(r, L)+O(1) .
$$

In the case where $f$ does not separate fibers of $\pi: X \rightarrow M$, we cannot estimate the growth of the ramification divisor in general. However, we have the following reduction theorem proved by J. Noguchi ([11, p. 272]):

TheOREm 1.5. (Noguchi) Let $f: X \rightarrow M$ be a meromorphic mapping. Then there exist a finite analytic covering space $\varpi: \underline{X} \rightarrow \mathbb{C}^{m}$, a surjective proper holomorphic mapping $\lambda: X \rightarrow \underline{X}$ and a meromorphic mapping $\underline{f}: \underline{X} \rightarrow M$ separating the fibers of $\varpi: \underline{X} \rightarrow \mathbb{C}^{m}$ such that the following $\overline{\text { diagram }}$

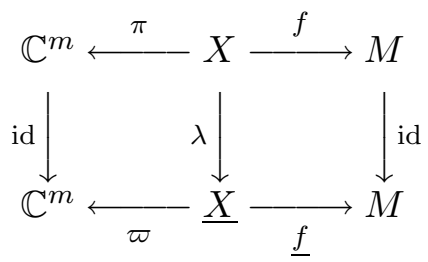

is commutative. Furthermore, if $f$ is dominant, so is $\underline{f}$.

From the above theorem, we can determine the proper domain of existence for an arbitrary meromorphic mapping $f: X \rightarrow M$. For the theory of algebroid reduction, see also [20].

Remark 1.6. We note that $\underline{X}$ is also a normal complex space. By making use of Theorem 1.4, we can easily obtain the following equalities (cf. [11, p. 273]):

$$
T_{f}(r, L)=T_{\underline{f}}(r, L), \quad \text { and } \quad N\left(r, f^{*} D\right)=N\left(r, \underline{f}^{*} D\right) .
$$

Thus we also have

$$
N_{k}\left(r, f^{*} D\right)=N_{k}\left(r, \underline{f}^{*} D\right)
$$


for each positive integer $k$. Hence, by Theorems 1.2 and 1.4, we have the following: For an arbitrary dominant meromorphic mapping $f: X \rightarrow M$, the following inequality

$$
q T_{f}(r, L)+T_{f}\left(r, K_{M}\right) \leq \sum_{j=1}^{q} N_{1}\left(r, f^{*} D_{j}\right)+N(r, \underline{B})+S_{f}(r)
$$

holds, where $\underline{B}$ is the ramification divisor of $\varpi: \underline{X} \rightarrow \mathbb{C}^{m}$. We also see that the following inequality holds:

$$
N(r, \underline{B}) \leq 2 \mu_{0}\left(s_{0}-1\right) T_{f}(r, L)+O(1) .
$$

Therefore we can apply Theorems 1.2 and 1.4 for an arbitrary meromorphic mapping $f: X \rightarrow M$. This observation is very useful and will be essentially used in the next section.

We finally give the defect relation and the ramification theorem for $f: X \rightarrow M$, which are needed later. For a line bundle $F$ over $M$, we define $[F / L]$ by

$$
[F / L]=\inf \left\{\gamma \in \mathbb{Q} ; \gamma L \otimes F^{-1} \text { is big }\right\} .
$$

Note that $[F / L]<0$ if and only if $F^{-1}$ is big. This is an easy consequence of Kodaira's lemma. We also set

$$
\Theta_{f}(D)=1-\limsup _{r \rightarrow+\infty} \frac{N_{1}\left(r, f^{*} D\right)}{T_{f}(r, L)} .
$$

Then Theorems 1.2 and 1.4 yield the following defect relation:

Theorem 1.7. (Defect Relation) Let $f$ and $D_{j}$ be as in Theorem 1.2. Suppose that $f$ separates the fibers of $\pi: X \rightarrow \mathbb{C}^{m}$. Then

$$
\sum_{j=1}^{q} \Theta_{f}\left(D_{j}\right) \leq\left[K_{M}^{-1} / L\right]+2 \mu_{0}\left(s_{0}-1\right) .
$$

Definition 1.8. Let $\nu$ be a positive integer and $D \in|L|$. A nonconstant holomorphic mapping $f: X \rightarrow M$ is ramified to order at least $\nu$ over $D$ if

$$
f^{*} D \geq \nu \operatorname{Supp} f^{*} D
$$

In the case $\operatorname{Supp} f^{*} D=\emptyset$, we may say that $f$ is ramified to order $+\infty$. If $\nu \geq 2$, then $D$ is said to be a totally ramified divisor for $f$. 
Now, by Theorems 1.2 and 1.4, we have the following ramification theorem:

Theorem 1.9. (Ramification Theorem) Let $f$ and $D_{j}$ be as in Theorem 1.2. Suppose that $f$ is ramified to order at least $\nu_{j}$ over $D_{j}(1 \leq j \leq q)$. Then

$$
\sum_{j=1}^{q}\left(1-\frac{1}{\nu_{j}}\right) \leq\left[K_{M}^{-1} / L\right]+2 \mu_{0}\left(s_{0}-1\right) .
$$

The following corollary directly follows from Theorem 1.9:

COROllary 1.10. The number of totally ramified divisors $D \in|L|$ for $f$ does not exceed $2\left[K_{M}^{-1} / L\right]+4 \mu_{0}\left(s_{0}-1\right)$.

Remark 1.11. Let $f$ and $D_{j}$ be as in Theorem 1.2. Suppose that

$$
q>2\left[K_{M}^{-1} / L\right]+4 \mu_{0}\left(s_{0}-1\right) .
$$

By the above corollary, we see that $\operatorname{Supp}_{1} f^{*} D_{j}$ is not empty for at least one $j$.

\section{§2. Criteria for algebraic dependence}

In this section we prove theorems on the propagation of algebraic dependence for some families of dominant meromorphic mappings from $X$ into a projective algebraic manifold $M$. We first give a definition of algebraic dependence of meromorphic mappings. Let $l$ be a positive integer not less than two. A proper algebraic subset $\Sigma$ of $M^{l}$ is said to be decomposable if, for some positive integer $s$ not grater than $l$, there exist positive integers $l_{1}, \ldots, l_{s}$ with $l=l_{1}+\cdots+l_{s}$ and algebraic subsets $\Sigma_{j} \subseteq M^{l_{j}}$ such that $\Sigma=\Sigma_{1} \times \cdots \times \Sigma_{s}$. In the case where $\Sigma$ is not decomposable, we say that $\Sigma$ is indecomposable. For meromorphic mappings $f_{1}, \ldots, f_{l}: X \rightarrow M$, we define a meromorphic mapping $f_{1} \times \cdots \times f_{l}: X \rightarrow M^{l}$ by

$$
\left(f_{1} \times \cdots \times f_{l}\right)(z)=\left(f_{1}(z), \ldots, f_{l}(z)\right), \quad z \in X-\left(I\left(f_{1}\right) \cup \cdots \cup I\left(f_{l}\right)\right),
$$

where $I\left(f_{j}\right)$ are the indeterminacy loci of $f_{j}$.

Definition 2.1. Let $S$ be an analytic subset of $X$. Nonconstant meromorphic mappings $f_{1}, \ldots, f_{l}: X \rightarrow M$ are said to be algebraically dependent on $S$ if there exists a proper algebraic subset $\Sigma$ of $M^{l}$ such that $\left(f_{1} \times \cdots \times f_{l}\right)(S) \subseteq \Sigma$ and $\Sigma$ is indecomposable. In this case, we also say that $f_{1}, \ldots, f_{l}$ are $\Sigma$-related on $S$. 
Let $L \rightarrow M$ be an ample line bundle. Let $D_{1}, \ldots, D_{q}$ be divisors in $|L|$ such that $D_{1}+\cdots+D_{q}$ has only simple normal crossings. Let $Z$ be an effective divisor on $X$, and let $k$ be a positive integer. If $Z=\sum_{j} \nu_{j} Z_{j}$ for distinct irreducible hypersurfaces $Z_{j}$ in $X$ and for nonnegative integers $\nu_{j}$, then we define the support of $Z$ with order at most $k$ by

$$
\operatorname{Supp}_{k} Z=\bigcup_{0<\nu_{j} \leq k} Z_{j}
$$

Let $S_{1}, \ldots, S_{q}$ be hypersurfaces in $X$ such that $\operatorname{dim} S_{i} \cap S_{j} \leq m-2$ for $i$ and $j(i \neq j)$, and let $k_{1}, \ldots, k_{q}$ be fixed positive integers. Assume that there exists at least one dominant meromorphic mapping $f_{0}: X \rightarrow M$ such that $\operatorname{Supp}_{k_{j}} f_{0}^{*} D_{j}=S_{j}$ for all $j$ with $1 \leq j \leq q$. As a matter of convenience, $f_{0}$ separates the fibers of $\pi: X \rightarrow M$. We denote by

$$
\mathscr{F}=\mathscr{F}\left(f_{0} ;\left\{k_{j}\right\} ;\left(X,\left\{S_{j}\right\}\right),\left(M,\left\{D_{j}\right\}\right)\right)
$$

the set of all dominant meromorphic mappings $f: X \rightarrow M$ with

$$
\operatorname{Supp}_{k_{j}} f^{*} D_{j}=S_{j}
$$

for all $j$ with $1 \leq j \leq q$. Let $F_{1}, \ldots, F_{l}$ be big line bundles over $M$. We define a line bundle $\widetilde{\widetilde{F}}$ over $M^{l}$ by

$$
\widetilde{F}=\pi_{1}^{*} F_{1} \otimes \cdots \otimes \pi_{l}^{*} F_{l},
$$

where $\pi_{j}: M^{l} \rightarrow M$ are the natural projections on the $j$-th factor. Let $\widetilde{L}$ be a big line bundle over $M^{l}$. Note that, in general,

$$
\widetilde{L} \notin \pi_{1}^{*} \operatorname{Pic}(M) \oplus \cdots \oplus \pi_{l}^{*} \operatorname{Pic}(M) .
$$

In the case of $\widetilde{L} \neq \widetilde{F}$, we assume that there exists a positive rational number $\tilde{\gamma}$ such that $\tilde{\gamma} \widetilde{F} \otimes \widetilde{L}^{-1}$ is big. If $\widetilde{L}=\widetilde{F}$, then we take $\tilde{\gamma}=1$. Let $\mathscr{R}$ be the set of all hypersurfaces $\Sigma$ in $X$ such that $\Sigma=\operatorname{Supp} \widetilde{D}$ for some $\widetilde{D} \in|\widetilde{L}|$ and $\Sigma$ is not decomposable. We assume that there exists a line bundle, say $F_{0}$, in $\left\{F_{1}, \ldots, F_{l}\right\}$ such that $F_{0} \otimes F_{j}^{-1}$ is either big or trivial for all $j$. Set $k_{0}=\max \left\{k_{1}, \ldots, k_{q}\right\}$. Since $L$ is ample, there exist a positive integer $\mu$ and a pair of sections $\sigma_{0}, \sigma_{1} \in H^{0}(M, \mu L)$ such that a meromorphic function $f^{*}\left(\sigma_{0} / \sigma_{1}\right)$ separates the fibers of $\pi: X \rightarrow \mathbb{C}^{m}$ for all such mappings $f$. 
We denote by $\mu_{0}$ the least positive integer among those $\mu$ 's. We define $L_{0} \in \operatorname{Pic}(M) \otimes \mathbb{Q}$ by

$$
L_{0}=\left(\sum_{j=1}^{q} \frac{k_{j}}{k_{j}+1}-2 \mu_{0}\left(s_{0}-1\right)\right) L \otimes\left(-\frac{\tilde{\gamma} l k_{0}}{k_{0}+1} F_{0}\right) .
$$

The following theorem is just the theorem stated in the Introduction.

THEOREM 2.2. Let $f_{1}, \ldots, f_{l}$ be arbitrary mappings in $\mathscr{F}$ and let $\Sigma$ be in $\mathscr{R}$. Suppose that $f_{1}, \ldots, f_{l}$ are $\Sigma$-related on $S$ and that $L_{0} \otimes K_{M}$ is big. Then $f_{1}, \ldots, f_{l}$ are $\Sigma$-related on $X$.

For the proof of Theorem 2.2, we need some lemmas. The following lemma is well-known as Kodaira's lemma:

LEMMA 2.3. (Kodaira) Let $L_{1}$ be an arbitrary line bundle over $M$. If $L$ is big, then there exists a positive integer $\mu$ such that $\mu L \otimes L_{1}$ is big and

$$
H^{0}\left(M, \nu L \otimes L_{1}\right) \neq\{0\}
$$

for all sufficiently big positive integers $\nu$.

For a proof, see, e.g., [9, Lemma 2].

Lemma 2.4. Let $F$ be a big line bundle over $M$. Let $f: X \rightarrow M$ be a dominant meromorphic mapping. If $\mu F \otimes L^{-1}$ is big for a positive integer $\mu$, then

$$
T_{f}(r, L) \leq \mu T_{f}(r, F)+O(1) .
$$

Proof. Since the line bundle $\mu F \otimes L^{-1}$ is big, we see that there exists a nonzero holomorphic section $\tau \in H^{0}\left(M, \nu\left(\mu F \otimes L^{-1}\right)\right)$ for a sufficiently large positive integer $\nu$. By Theorem 1.1, we have

$$
\begin{aligned}
N\left(r, f^{*}(\tau)\right) & \leq T_{f}\left(r, \nu\left(\mu\left(F \otimes L^{-1}\right)\right)+O(1)\right. \\
& =\mu \nu T_{f}(r, F)-\nu T_{f}(r, L)+O(1) .
\end{aligned}
$$

This shows the desired conclusion.

Lemma 2.5. Let $f$ be an arbitrary mapping in $\mathscr{F}$. Then

$$
\begin{aligned}
& q T_{f}(r, L)+T_{f}\left(r, K_{M}\right) \\
& \quad \leq \frac{k_{0}}{k_{0}+1} N(r, S)+\sum_{j=1}^{q} \frac{1}{k_{j}+1} N\left(r, f^{*} D_{j}\right)+N(r, B)+S_{f}(r) .
\end{aligned}
$$


Proof. Since $k_{j} /\left(k_{j}+1\right) \leq k_{0} /\left(k_{0}+1\right)$ for all $j$ with $1 \leq j \leq q$, we easily see

$$
N_{1}\left(r, f^{*} D_{j}\right) \leq \frac{k_{0}}{k_{0}+1} N\left(r, S_{j}\right)+\frac{1}{k_{j}+1} N\left(r, f^{*} D_{j}\right) .
$$

Hence, by Theorem 1.2 and $\operatorname{dim} S_{i} \cap S_{j} \leq m-2$ for $i \neq j$, we have our assertion.

For brevity, set $\tilde{f}=f_{1} \times \cdots \times f_{l}$.

LemMA 2.6. Let $f_{1}, \ldots, f_{l} \in \mathscr{F}$. Suppose that $\tilde{f}(S) \subseteq \Sigma$ and $\tilde{f}(X) \nsubseteq$ $\Sigma$ for some $\Sigma \in \mathscr{R}$. Then

$$
N(r, S) \leq \tilde{\gamma} \sum_{j=1}^{l} T_{f_{j}}\left(r, F_{j}\right)+O(1)
$$

In particular, the following two inequalities hold:

$$
\begin{aligned}
& N(r, S) \leq \tilde{\gamma} \sum_{j=1}^{l}\left[F_{j} / L\right] T_{f_{j}}(r, L)+O(1) \quad \text { and } \\
& N(r, S) \leq \tilde{\gamma} \sum_{j=1}^{l} T_{f_{j}}\left(r, F_{0}\right)+O(1)
\end{aligned}
$$

Proof. Take a divisor $\widetilde{D} \in|\widetilde{L}|$ so that $\Sigma=\operatorname{Supp} \widetilde{D}$. Then, by Theorem 1.1 , we see

$$
\begin{aligned}
N(r, S) & \leq N\left(r, \tilde{f}^{*} \widetilde{D}\right) \\
& \leq T_{\widetilde{f}}(r, \widetilde{L})+O(1) \\
& \leq \tilde{\gamma} T_{\widetilde{f}}(r, \widetilde{F})+O(1) \\
& \leq \tilde{\gamma} \sum_{j=1}^{l} T_{f_{j}}\left(r, F_{j}\right)+O(1) .
\end{aligned}
$$

Thus we have our assertion.

We now give the proof of Theorem 2.2. For brevity, we set

$$
S(r)=\sum_{j=1}^{l} S_{f_{j}}(r) \quad \text { and } \quad T(r, F)=\sum_{j=1}^{l} T_{f_{j}}(r, F)
$$

for $F \in \operatorname{Pic}(M) \otimes \mathbb{Q}$. 
Proof of Theorem 2.2. By Remark 1.6, we may assume that all $f_{j}$ separate the fibers of $\pi: X \rightarrow \mathbb{C}^{m}$. Suppose that $\tilde{f}(X) \nsubseteq \Sigma$. By Theorem 1.1, Lemmas 2.5 and 2.6, we have

$$
\begin{aligned}
\sum_{j=1}^{q}\left(\frac{k_{j}}{k_{j}+1}\right) T_{f_{i}}(r, L)+T_{f_{i}}\left(r, K_{M}\right) & \leq \frac{k_{0}}{k_{0}+1} N(r, S)+N(r, B)+S_{f_{i}}(r) \\
& \leq \frac{\tilde{\gamma} k_{0}}{k_{0}+1} T\left(r, F_{0}\right)+N(r, B)+S_{f_{i}}(r)
\end{aligned}
$$

for all $i$. Hence we have

$$
\left(\sum_{j=1}^{q} \frac{k_{j}}{k_{j}+1}\right) T(r, L)+T\left(r, K_{M}\right) \leq \frac{\tilde{\gamma} l k_{0}}{k_{0}+1} T\left(r, F_{0}\right)+l N(r, B)+S(r) .
$$

Therefore we see

$$
T\left(r, L_{0}\right)+T\left(r, K_{M}\right) \leq S(r) .
$$

Since $L_{0} \otimes K_{M}$ is big, there exists a positive integer $\mu$ such that $\mu\left(L_{0} \otimes\right.$ $\left.K_{M}\right) \otimes L^{-1}$ is big. Thus, by Lemma 2.4 , we get

$$
T(r, L) \leq \mu\left(T\left(r, L_{0}\right)+T\left(r, K_{M}\right)\right)+O(1) .
$$

This gives a contradiction. Therefore, we have $\tilde{f}(X) \subseteq \Sigma$. We have now completed the proof of Theorem 2.2.

As was stated in the above proof, under our assumptions, we may assume that all meromorphic mappings $f_{j}: X \rightarrow M$ separate the fiber of $X \rightarrow \mathbb{C}^{m}$. This comes from Noguchi's theorem. Thus we assume this in what follows.

Set $\gamma_{0}=\left[L_{0}^{-1} \otimes K_{M}^{-1} / L\right]$. Under the condition that $L_{0} \otimes K_{M}$ is big, we see $\gamma_{0}<0$. In the case where $\gamma_{0}$ is nonnegative, we cannot conclude the propagation of algebraic dependence in general. In the case of a positive $\gamma_{0}$, the propagation of algebraic dependence does not occur even if we assume the existence of Picard's deficient divisors (see Remarks 2.14 below). However, in the case of $\gamma_{0}=0$, we have the following theorem on the propagation of algebraic dependence under a condition on the existence of Nevanlinna's deficient divisors. This shows that the existence of such a divisor gives strong effect on the uniqueness problem of meromorphic mappings. 
Theorem 2.7. Let $f_{1}, \ldots, f_{l}$ be as in Theorem 2.2. Suppose that $f_{0}=$ $f_{k}$ for some $k$ with $1 \leq k \leq q$ and that $\left[L_{0}^{-1} \otimes K_{M}^{-1} / L\right]=0$. If $\delta_{f_{0}}\left(D_{j}\right)>0$ for at least one $j$ with $1 \leq j \leq q$, then $f_{1}, \ldots, f_{l}$ are $\Sigma$-related on $X$.

The following lemma is a crucial step for the proof of Theorem 2.7:

Lemma 2.8. Let $f_{1}, \ldots, f_{l}$ be as in Theorem 2.7. If $\left[L_{0}^{-1} \otimes K_{M}^{-1} / L\right]$ $=0$ and $\tilde{f}(X) \nsubseteq \Sigma$, then there exist positive constants $C_{1}$ and $C_{2}$ such that

$$
C_{1} \leq \frac{T_{f_{j}}(r, L)}{T_{f_{1}}(r, L)} \leq C_{2}
$$

for all sufficiently large $r$ with $r \notin I$, where $I$ is an exceptional set for $S(r)$.

Proof. We first show the following: For any positive rational number $\nu$ with $\tilde{\gamma} \nu<1$, we have that

$$
\nu \tilde{\gamma} T\left(r, F_{0}\right)+O(1)<N(r, S)
$$

for all sufficiently large $r \notin I$. Assume the contrary. Then there exist a positive rational number $\nu_{0}$ with $\tilde{\gamma} \nu_{0}<1$ and a monotone increasing sequence $\left\{r_{k}\right\}$ with $r_{k} \notin I$ such that $r_{k} \rightarrow+\infty$ and

$$
N\left(r_{k}, S\right) \leq \nu_{0} \tilde{\gamma} T\left(r_{k}, F_{0}\right)+O(1) .
$$

By Lemmas 2.5 and 2.6, we have

$$
\begin{aligned}
& \left(\sum_{j=1}^{q} \frac{k_{j}}{k_{j}+1}\right) T\left(r_{k}, L\right)+T\left(r_{k}, K_{M}\right) \\
& \quad \leq\left(\frac{l k_{0}}{k_{0}+1}\right) \nu_{0} \tilde{\gamma} T\left(r_{k}, F_{0}\right)+l N\left(r_{k}, B\right)+S\left(r_{k}\right) .
\end{aligned}
$$

We define a positive rational number $\mu_{1}$ by

$$
\mu_{1}=\frac{k_{0}\left(1-\nu_{0} \tilde{\gamma}\right)}{k_{0}+1}
$$

Then we have

$$
T\left(r_{k}, L_{0} \otimes K_{M}\right)+\mu_{1} T\left(r_{k}, F_{0}\right) \leq S\left(r_{k}\right) .
$$


It follows from $\left[L_{0}^{-1} \otimes K_{M}^{-1} / L\right]=0$ that $L_{0} \otimes K_{M} \otimes \mu_{1} F_{0}$ is big. Hence there exists a positive constant $C$ such that

$$
C T\left(r_{k}, L\right) \leq T\left(r_{k}, L_{0}\right)+T\left(r_{k}, K_{M}\right)+\mu_{1} T\left(r_{k}, F_{0}\right)+O(1) .
$$

Thus we see $C T\left(r_{k}, L\right) \leq S\left(r_{k}\right)$. This shows $C \leq 0$, which is absurd. Hence we have (2.9). Next we note that

$$
N(r, S) \leq q T_{f_{1}}(r, L)+O(1) .
$$

This is an immediate consequence of Theorem 1.1. We now have

$$
\mu T(r, L) \leq q T_{f_{1}}(r, L)+O(1),
$$

where $\mu=\nu \tilde{\gamma}$. Note that $0<\mu<1$. This completes the proof of Lemma 2.8.

Proof of Theorem 2.7. For the proof of Theorem 2.7, it suffices to show that $\tilde{f}(X) \subseteq \Sigma$. Assume the contrary. We may assume that $f_{0}=f_{1}$. By the definition of Nevanlinna's deficiency, for any $\varepsilon>0$, there exists $r_{0}>0$ such that

$$
N\left(r, f_{k}^{*} D_{j}\right)<\left(1-\delta_{f_{k}}\left(D_{j}\right)+\varepsilon\right) T_{f_{k}}(r, L)
$$

for all $r \geq r_{0}(r \notin I)$, where $I \subseteq[1,+\infty)$ is a Borel subset with finite measure. We may assume that the exceptional set for $S(r)$ is included in I. By Lemmas 2.5 and 2.6, we have

$$
\begin{aligned}
T\left(r, L_{0}\right)+T\left(r, K_{M}\right) & \leq-\sum_{k=0}^{p} \sum_{j=1}^{q} \frac{1}{k_{j}+1}\left(\delta_{f_{k}}\left(D_{j}\right)-\varepsilon\right) T_{f_{k}}(r, L)+S(r) \\
& \leq q \varepsilon T(r, L)-\frac{1}{k_{1}+1} \delta_{f_{1}}\left(D_{1}\right) T_{f_{1}}(r, L)+S(r) .
\end{aligned}
$$

Hence

$$
\frac{1}{k_{1}+1} \delta_{f_{1}}\left(D_{1}\right) T_{f_{1}}(r, L)+T\left(r, L_{0}\right)+T\left(r, K_{M}\right)-q \varepsilon T(r, L) \leq S(r) .
$$

Take a rational number $\gamma>0$. It follows from $\left[L_{0}^{-1} \otimes K_{M}^{-1} / L\right]=0$ that

$$
-\gamma T(r, L) \leq T\left(r, L_{0}\right)+T\left(r, K_{M}\right)+O(1)
$$

and hence

$$
\frac{1}{k_{1}+1} \delta_{f_{1}}\left(D_{1}\right) T_{f_{1}}(r, L)-(\gamma+q \varepsilon) T(r, L) \leq S(r) .
$$

By Lemma 2.8, we see $\delta_{f_{1}}\left(D_{1}\right)=0$, which contradicts the assumption. Thus we have now completed the proof of Theorem 2.7. 
We now give a criteria for the propagation of dependence, which is an immediate corollary of the proof of Theorem 2.2 (cf. [5] and [21]). Set

$$
p_{0}=\sum_{j=1}^{q} \frac{k_{j}}{k_{j}+1}-\left[K_{M}^{-1} / L\right]-2 \mu_{0}\left(s_{0}-1\right)
$$

We also set

$$
m_{1}=q-\left[K_{M}^{-1} / L\right]-2 \mu_{0}\left(s_{0}-1\right) \quad \text { and } \quad m_{j}=q-\left[K_{M}^{-1} / L\right](2 \leq j \leq l) .
$$

Then we have the following:

Proposition 2.10. Let $f_{1}, \ldots, f_{l} \in \mathscr{F}$. Suppose that they are $\Sigma$ related on $S$. If all $m_{j}$ are positive and if

$$
p_{0}-\frac{\tilde{\gamma} l k_{0}}{k_{0}+1}\left[F_{1} / L\right]+m_{1} \sum_{j=2}^{l}\left(p_{0}-\frac{\tilde{\gamma} l k_{0}}{k_{0}+1}\left[F_{j} / L\right]\right)>0,
$$

then $f_{1}, \ldots, f_{l}$ are $\Sigma$-related on $X$.

Proof. We note that

$$
-T_{f}\left(r, K_{M}\right) \leq\left[K_{M}^{-1} / L\right] T_{f}(r, L)+O(1)
$$

and hence

$$
\left(q-\left[K_{M}^{-1} / L\right]\right) T_{f}(r, L) \leq \sum_{j=1}^{q} N_{1}\left(r, f^{*} D_{j}\right)+N(r, B)+S_{f}(r) .
$$

We first show

$$
m_{1} T_{f_{1}}(r, L) \leq T_{f_{j}}(r, L)+S_{f_{1}}(r)
$$

for all $j$. By Theorems 1.1 and 1.2, we see

$$
\begin{aligned}
q T_{f_{1}}(r, L)+T_{f_{1}}\left(r, K_{M}\right) & \leq N(r, Z)+N(r, B)+S_{f_{1}}(r) \\
& \leq T_{f_{j}}(r, L)+N(r, B)+S_{f_{1}}(r) \\
& \leq T_{f_{j}}(r, L)+2 \mu_{0}\left(s_{0}-1\right) T_{f_{1}}(r, L)+S_{f_{1}}(r)
\end{aligned}
$$

which gives (2.11). We also have

$$
m_{j} T_{f_{j}}(r, L) \leq T_{f_{1}}(r, L)+N(r, B)+S_{f_{j}}(r)
$$


for $j(\neq 1)$ in the same way, and hence

$$
m_{j} T_{f_{j}}(r, L) \leq\left(2 \mu_{0}\left(s_{0}-1\right)+1\right) T_{f_{1}}(r, L)+S_{f_{j}}(r) .
$$

The inequalities (2.11) and (2.12) yield

$$
T_{f_{1}}(r, L) \sim T_{f_{j}}(r, L) \quad \text { as } \quad r \rightarrow+\infty .
$$

As in the proof of Theorem 2.2, we have

$$
\left(\sum_{j=1}^{q} \frac{k_{j}}{k_{j}+1}-\left[K_{M}^{-1} / L\right]\right) T_{f_{i}}(r, L) \leq \frac{k_{0}}{k_{0}+1} N(r, S)+N(r, B)+S_{f_{i}}(r)
$$

for all $i$. Hence, by Lemmas 2.4 and 2.6, we have

$$
p_{0} T(r, L) \leq \frac{\tilde{\gamma} l k_{0}}{1+k_{0}} \sum_{j=1}^{l}\left[F_{j} / L\right] T_{f_{j}}(r, L)+2 l \mu_{0}\left(s_{0}-1\right) T_{f_{1}}(r, L)+S(r) .
$$

By (2.11), we get

$$
\left(p_{0}-\frac{\tilde{\gamma} l k_{0}}{k_{0}+1}\left[F_{1} / L\right]+m_{1} \sum_{j=2}^{l}\left(p_{0}-\frac{\tilde{\gamma} l k_{0}}{k_{0}+1}\left[F_{j} / L\right]\right)\right) T_{f_{1}}(r, L) \leq S(r) .
$$

By the assumption in this proposition and (2.13), we have a contradiction.

Remarks 2.14. (1) In the past, the propagation of dependence has been studied under conditions on the growth of mappings and the ramification divisor $B$ of $\pi: X \rightarrow \mathbb{C}^{m}$. For example, W. Stoll proved some interesting theorems on the propagation of dependence of meromorphic mappings $f$ : $X \rightarrow M$ under a condition on the growth of mappings in different settings (cf. [21]). In his results, at least one of the mappings $f_{j}$ must grow quicker than the ramification divisor $B$, that is, it is assumed that

$$
\lim _{r \rightarrow+\infty} \frac{N(r, B)}{T_{f}(r, L)}=0 .
$$

However, the existence of such a mapping is a delicate matter even if $X$ and $M$ are Riemann surfaces (cf. L. Sario [13]). In [21], he already declared that his condition can be replaced by a condition on the existence of mappings separating the fibers of $\pi: X \rightarrow \mathbb{C}^{m}$ in some cases. 
(2) If $\left[L_{0}^{-1} \otimes K_{M}^{-1} / L\right]$ is positive, we cannot conclude the propagation of dependence under the condition on the existence of deficient divisors. We now give an example. Let $M=\mathbb{P}_{1}(\mathbb{C})$ and $l=2$. Suppose that $H \rightarrow \mathbb{P}_{1}(\mathbb{C})$ is the hyperplane bundle. Let $L=H$ and $F_{1}=F_{2}=H$. We now take

$$
\widetilde{L}=\widetilde{F}=\pi_{1}^{*} H \otimes \pi_{2}^{*} H .
$$

Let $f_{0}: \mathbb{C} \rightarrow \mathbb{P}_{1}(\mathbb{C})$ be a meromorphic function defined by $f_{0}(z)=\exp z$. Set $D_{1}=0, D_{2}=\infty, D_{3}=1$ and $D_{4}=-1$. Then it is clear that $D_{1}$ and $D_{2}$ are Picard's deficient values of $f_{0}$. Let $k_{j}=1$ for all $j$ and put $S_{j}=\operatorname{Supp}_{1} f_{0}^{*} D_{j}$. Now, we see that $\left[L_{0}^{-1} \otimes K_{\mathbb{P}_{1}(\mathbb{C})}^{-1} / L\right]=1$ and $f_{1}(z)=$ $\exp (-z) \in \mathscr{F}$. If $\Sigma$ is the diagonal of $\mathbb{P}_{1}(\mathbb{C})^{2}$, then $\Sigma \in \mathscr{R}$. It is clear that $\left(f_{0} \times f_{1}\right)(S) \subseteq \Sigma$ and $\left(f_{0} \times f_{1}\right)(\mathbb{C}) \nsubseteq \Sigma$. Note that the proofs of the above theorems also work in the case where some of $S_{j}$ are the empty sets.

(3) The case, where either all $k_{j}=1$ or all $k_{j}=+\infty$, are essentially important in our study. We now consider the case where $k_{j}=+\infty$ for some $j$. We first note that $\operatorname{Supp} f^{*} D=\operatorname{Supp}_{k_{j}} f^{*} D$ if $k_{j}=+\infty$. Set $k_{j} /\left(k_{j}+1\right)=1$ and $1 /\left(k_{j}+1\right)=0$ for $k_{j}=+\infty$. Then it is easy to see that the proof of Theorem 2.2 also works in the case where $k_{j}=+\infty$ for some $j$. In the case where $\left[L_{0}^{-1} \otimes K_{M}^{-1} / L\right]=0$, we have the same conclusion as in Theorem 2.7 if we assume that $\delta_{f_{0}}\left(D_{j}\right)>0$ with $k_{j} \neq+\infty$ for some $j$. The proof of Theorem 2.7 remains still valid under this condition. Note that the condition $k_{j} \neq+\infty$ cannot be dropped. Indeed, let $D_{j}, f_{j}$ and $\Sigma$ be as in (2). Now let $k_{j}=+\infty$ for all $1 \leq j \leq 4$. Then we see that $\left[L_{0}^{-1} \otimes K_{\mathbb{P}_{1}(\mathbb{C})}^{-1} / L\right]=0$ and $\left(f_{0} \times f_{1}\right)(S) \subseteq \Sigma$, but $\left(f_{0} \times f_{1}\right)(\mathbb{C}) \not \Sigma$.

(4) We consider the case where $D_{i}$ and $D_{j}$ are not linearly equivalent for some pair $(i, j)$ but all the Chern classes $c_{1}\left(\left[D_{j}\right]\right)$ are identical. In this case, by the proof of Theorem 2.2, we easily see that Theorem 2.2 remains valid provided that

$$
\left(\sum_{j=1}^{q} \frac{k_{j}}{k_{j}+1}-2 \mu_{0}\left(s_{0}-1\right)\right)\left[D_{1}\right] \otimes\left(-\frac{\tilde{\gamma} l k_{0}}{k_{0}+1} F_{0}\right)
$$

is ample. This fact is used lalter.

Now, let us consider a more general case. Let $L_{1}, \ldots, L_{l}$ be ample line bundles over $M$ and let $q_{1}, \ldots, q_{l}$ be positive integers. Assume that $D_{j}=D_{j 1}+\cdots+D_{j q_{j}} \in\left|q_{j} L_{j}\right|$ has only normal crossings, where $D_{j k} \in|L|$. Let $Z$ be a hypersurface in $X$. We denote by

$$
\mathscr{G}=\mathscr{G}\left(\left\{k_{j}\right\} ;(X, Z) ;\left(M,\left\{D_{j}\right\}\right)\right)
$$


the family of dominant meromorphic mappings $f: X \rightarrow M$ such that

$$
\operatorname{Supp}_{k_{j}} f^{*} D_{j}=Z
$$

for some $j$ with $1 \leq j \leq l$. In the case where all $L_{j}$ are identical (say $L$ ), we define $G_{0} \in \operatorname{Pic}(M) \otimes \mathbb{Q}$ by

$$
G_{0}=\left(\min _{1 \leq j \leq l}\left\{\frac{q_{j} k_{j}}{k_{j}+1}\right\}-2 \mu_{0}\left(s_{0}-1\right)\right) L \otimes\left(-\frac{\tilde{\gamma} l k_{0}}{k_{0}+1} F_{0}\right) .
$$

Then the following theorem is fundamental in this case:

TheOREM 2.15. Let $f_{1}, \ldots, f_{l}$ be arbitrary mappings in $\mathscr{G}$ and let $\Sigma \in$ $\mathscr{R}$. Suppose that $f_{1}, \ldots, f_{l}$ are $\Sigma$-related on $Z$. If $G_{0} \otimes K_{M}$ is big, then $f_{1}, \ldots, f_{l}$ are $\Sigma$-related on $X$.

Proof. Suppose that $\tilde{f}(X) \nsubseteq \Sigma$. Without loss of generality, we may assume that $\operatorname{Supp}_{k_{j}} f_{j}^{*} D_{j}=Z$ for all $j$. We first note that the following inequality holds (cf. Lemmas 2.5):

$$
\begin{aligned}
& q_{j} T_{f_{j}}(r, L)+T_{f_{j}}\left(r, K_{M}\right) \\
& \quad \leq \frac{k_{0}}{k_{0}+1} N(r, Z)+\frac{1}{k_{j}+1} N\left(r, f_{j}^{*} D_{j}\right)+N(r, B)+S_{f_{j}}(r) .
\end{aligned}
$$

The following inequality also remains valid in this case:

$$
N(r, Z) \leq \tilde{\gamma} \sum_{j=1}^{l} T_{f_{j}}\left(r, F_{j}\right)+O(1)
$$

Thus we get

$$
\frac{q_{j} k_{j}}{k_{j}+1} T_{f_{j}}(r, L)+T_{f_{j}}\left(r, K_{M}\right) \leq\left(\frac{k_{0}}{k_{0}+1}\right) \tilde{\gamma} T\left(r, F_{0}\right)+N(r, B)+S_{f_{j}}(r) .
$$

So we have

$$
\begin{aligned}
& \left(\min _{1 \leq j \leq l}\left\{\frac{q_{j} k_{j}}{k_{j}+1}\right\}\right) T(r, L)+T\left(r, K_{M}\right) \\
& \quad \leq\left(\frac{\tilde{\gamma} l k_{0}}{k_{0}+1}\right) T\left(r, F_{0}\right)+l N(r, B)+S(r) .
\end{aligned}
$$

Hence we see

$$
T\left(r, G_{0}\right)+T\left(r, K_{M}\right) \leq S(r) .
$$

Since $G_{0} \otimes K_{M}$ is big, this gives a contradiction. 
We now return the general case. We note the following: In the case where $L_{i}$ and $L_{j}$ are not same for some $i$ and $j$ but all the Chern classes $c_{1}\left(L_{j}\right)$ are identical, the conclusion of Theorem 2.15 is atill valid if the line bundle

$$
\left(\min _{1 \leq j \leq l}\left\{\frac{q_{j} k_{j}}{k_{j}+1}\right\}-2 \mu_{0}\left(s_{0}-1\right)\right) L_{1} \otimes\left(-\frac{\tilde{\gamma} l k_{0}}{k_{0}+1} F_{0}\right)
$$

is ample. We give two criteria for the propagation of dependence similar to Proposition 2.10, which are corollaries of the proof of Theorem 2.15. Set

$$
n_{1}=q_{1}-\left[K_{M}^{-1} / L_{1}\right]-2 \mu_{0}\left(s_{0}-1\right) \quad \text { and } \quad n_{j}=q_{j}-\left[K_{M}^{-1} / L_{j}\right](2 \leq j \leq l) .
$$

We also set

$$
p_{j}=\frac{q_{j} k_{j}}{1+k_{j}}-\left[K_{M}^{-1} / L_{j}\right]-2 \mu_{0}\left(s_{0}-1\right)
$$

for all $j$ with $1 \leq j \leq l$. Then we have the following result:

Proposition 2.16. Let $f_{1}, \ldots, f_{l}$ be arbitrary mappings in $\mathscr{G}$ and $\Sigma \in$ $\mathscr{R}$. Suppose that $f_{1}, \ldots, f_{l}$ are $\Sigma$-related on $Z$. If $n_{j}>0$ for all $j$ and if

$$
p_{1}-\frac{\tilde{\gamma} l k_{0}}{k_{0}+1}\left[F_{1} / L_{1}\right]+n_{1} \sum_{j=2}^{l}\left(p_{j}-\frac{\tilde{\gamma} l k_{0}}{k_{0}+1}\left[F_{j} / L_{j}\right]\right)>0,
$$

then $f_{1}, \ldots, f_{l}$ are $\Sigma$-related on $X$.

Proof. As in the proof of Proposition 2.10, we get

$$
n_{1} T_{f_{1}}\left(r, L_{1}\right) \leq T_{f_{j}}\left(r, L_{j}\right)+S_{f_{1}}(r)
$$

for all $j$, and

$$
n_{j} T_{f_{j}}\left(r, L_{j}\right) \leq\left(2 \mu_{0}\left(s_{0}-1\right)+1\right) T_{f_{1}}\left(r, L_{1}\right)+S_{f_{j}}(r) .
$$

Hence $T_{f_{1}}\left(r, L_{1}\right) \sim T_{f_{j}}\left(r, L_{j}\right)$ as $r \rightarrow+\infty$. Next, we also have

$$
\begin{aligned}
& \sum_{j=1}^{l}\left(\frac{q_{j} k_{j}}{k_{j}+1}-\left[K_{M} / L_{j}\right]\right) T_{f_{j}}\left(r, L_{j}\right) \\
& \quad \leq\left(\frac{l k_{0}}{k_{0}+1}\right) N(r, Z)+l N(r, B)+S(r)
\end{aligned}
$$


It follows from

$$
N(r, Z) \leq \sum_{j=1}^{l} \tilde{\gamma}\left[F_{j} / L_{j}\right] T_{f_{j}}\left(r, L_{j}\right)+O(1)
$$

that

$$
\sum_{j=1}^{l}\left(\frac{q_{j} k_{j}}{k_{j}+1}-\left(\frac{\tilde{\gamma} l k_{0}}{k_{0}+1}\right)\left[K_{M} / L_{j}\right]\right) T_{f_{j}}\left(r, L_{j}\right)-2 l \mu_{0}\left(s_{0}-1\right) T_{f_{1}}\left(r, L_{1}\right) \leq S(r) .
$$

Therefore we see

$$
\left(p_{1}-\frac{\tilde{\gamma} l k_{0}}{k_{0}+1}\left[F_{1} / L_{1}\right]+n_{1} \sum_{j=2}^{l}\left(p_{j}-\frac{\tilde{\gamma} l k_{0}}{k_{0}+1}\left[F_{j} / L_{j}\right]\right)\right) T_{f_{1}}\left(r, L_{1}\right) \leq S(r) .
$$

This gives a contradiction. We now have completed the proof.

Set $e_{0}=2 \mu_{0}\left(s_{0}-1\right)+1$. By a method similar to the above proof, we have the following:

Proposition 2.17. Let $f_{1}, \ldots, f_{l}$ be as in Proposition 2.16. If $n_{j}>0$ for all $j$ and if

$$
p_{1}-\frac{\tilde{\gamma} l k_{0}}{k_{0}+1}\left[F_{1} / L_{1}\right]+\sum_{j=2}^{l}\left(n_{1} p_{j}-\frac{\tilde{\gamma} l e_{0} k_{0}}{n_{j}\left(k_{0}+1\right)}\left[F_{j} / L_{j}\right]\right)>0
$$

then $f_{1}, \ldots, f_{l}$ are $\Sigma$-related on $X$.

Proof. As in the proof of Proposition 2.16, we see that

$$
\begin{aligned}
& n_{1} T_{f_{1}}\left(r, L_{1}\right) \leq T_{f_{j}}\left(r, L_{j}\right)+S_{f_{1}}(r) \quad \text { and } \\
& n_{j} T_{f_{j}}\left(r, L_{j}\right) \leq e_{0} T_{f_{1}}\left(r, L_{1}\right)+S_{f_{j}}(r)
\end{aligned}
$$

for all $j$. On the other hand, we get

$$
\left(p_{1}-\frac{\tilde{\gamma} l k_{0}}{k_{0}+1}\left[F_{1} / L_{1}\right]\right) T_{f_{1}}\left(r, L_{1}\right)+\sum_{j=2}^{l}\left(p_{j}-\frac{\tilde{\gamma} l k_{0}}{k_{0}+1}\left[F_{j} / L_{j}\right]\right) T_{f_{j}}\left(r, L_{j}\right) \leq S(r) .
$$

Thus we see

$$
\left(p_{1}-\frac{\tilde{\gamma} l k_{0}}{k_{0}+1}\left[F_{1} / L_{1}\right]+\sum_{j=2}^{l}\left(n_{1} p_{j}-\frac{\tilde{\gamma} l e_{0} k_{0}}{n_{j}\left(k_{0}+1\right)}\left[F_{j} / L_{j}\right]\right)\right) T_{f_{1}}\left(r, L_{1}\right) \leq S(r) .
$$

This contradicts our assumption. Therefore we have the desired conclusion. 
As was mentioned in Remark 2.14 , the case where all $k_{j}=1$ is important from the viewpoint of Nevanlinna theory. Note that the conclusions of Propositions 2.10, 2.16 and 2.17 are still valid for the case where some of $k_{j}=+\infty$. Indeed, the proofs of Theorems 2.2 and 2.15 also work in this case, and hence we have our assertion. We also have a criterion in the case where $G_{0} \otimes K_{M}$ is not big. In this case, we can show the following theorem by an argument similar to the proof of Theorem 2.7:

THEOREM 2.18. Let $f_{1}, \ldots, f_{l}$ be arbitrary mappings in $\mathscr{G}$ and let $\Sigma \in$ $\mathscr{R}$. Suppose that $f_{1}, \ldots, f_{l}$ are $\Sigma$-related on $Z$ and $\left[G_{0}^{-1} \otimes K_{M}^{-1} / L\right]=0$. If there exists $f_{i}$ such that $\delta_{f_{i}}\left(D_{j}\right)>0$ for at least one $j$ with $1 \leq j \leq q$, then $f_{1}, \ldots, f_{l}$ are $\Sigma$-related on $X$.

To see what the criteria proved in this section amount to, we will give some applications and examples in the next three sections.

\section{§3. Unicity theorems for meromorphic mappings}

As we mentioned in the Introduction, a certain kind of unicity theorems are just theorems on propagation of dependence. In this section, we give some unicity theorems as an application of criteria for dependence by taking line bundles $F_{j}$ of special type. For the details of this direction, see, e.g, [2], [3], [4] and [18]. We keep the same notation as in Section 2. Let $\Phi: M \rightarrow \mathbb{P}_{n}(\mathbb{C})$ be a meromorphic mapping with $\operatorname{rank} \Phi=\operatorname{dim} M$. We denote by $H$ the hyperplane bundle over $\mathbb{P}_{n}(\mathbb{C})$. Now, let $l=2$ and take $F_{1}=F_{2}=\Phi^{*} H$. We also take $\widetilde{L}=\widetilde{F}$. Then we see

$$
L_{0}=\left(\sum_{j=1}^{q} \frac{k_{j}}{k_{j}+1}-2 \mu_{0}\left(s_{0}-1\right)\right) L \otimes\left(-\frac{2 k_{0}}{k_{0}+1} \Phi^{*} H\right) .
$$

A set $\left\{D_{j}\right\}_{j=1}^{q}$ of divisors is said to be generic with respect to $f_{0}$ and $\Phi$ provided that

$$
R_{j}:=f_{0}\left(X-I\left(f_{0}\right)\right) \cap \operatorname{Supp} D_{j} \cap\{w \in M: \operatorname{rank} d \Phi(w)=\operatorname{dim} M\} \neq \emptyset
$$

for at least one $j$ with $1 \leq j \leq q$. We assume that $\left\{D_{j}\right\}_{j=1}^{q}$ is generic with respect to $f_{0}$ and $\Phi$. Let $\mathscr{F}_{1}$ be the set of all mappings $f \in \mathscr{F}$ such that $f=f_{0}$ on $S$. Then we have the following unicity theorems (cf. [2]):

Theorem 3.1. (1) Suppose that $L_{0} \otimes K_{M}$ is big. Then the family $\mathscr{F}_{1}$ contains just one mapping $f_{0}$. 
(2) Suppose that $\left[L_{0}^{-1} \otimes K_{M}^{-1} / L\right]=0$. If $\delta_{f_{0}}\left(D_{j}\right)>0$ for at least one $j$ with $1 \leq j \leq q$, then the family $\mathscr{F}_{1}$ contains just one mapping $f_{0}$.

Proof. Let $f \in \mathscr{F}_{1}$. We denote by $\Delta$ the diagonal of $\mathbb{P}_{n}(\mathbb{C})^{2}$. Set $\Psi=\Phi \times \Phi$. Since $f=f_{0}$ on $S$, it is clear that $\Psi \circ\left(f \times f_{0}\right)(S) \subseteq \Delta$. Suppose that $\Phi \circ f \not \equiv \Phi \circ f_{0}$. Then we can take a hypersurface $\Sigma \in \mathscr{R}$ so that $\left(f \times f_{0}\right)(S) \subseteq \Sigma$ and $\left(f \times f_{0}\right)(X) \nsubseteq \Sigma$. Indeed, we define the line bundle $\widetilde{H} \rightarrow \mathbb{P}_{n}(\mathbb{C})^{2}$ by $\widetilde{H}=\pi_{1}^{*} H \otimes \pi_{2}^{*} H$. Note that $\widetilde{L}=\Psi^{*} \widetilde{H}$. We define a meromorphic mapping $\varphi: \mathbb{C}^{m} \rightarrow \mathbb{P}_{n}(\mathbb{C})^{2}$ by $\varphi=\Psi \circ\left(f \times f_{0}\right)$. Since $\Phi \circ f \not \equiv \Phi \circ f_{0}$, there exists a holomorphic section $\tilde{\sigma}$ of $\widetilde{H} \rightarrow \mathbb{P}_{n}(\mathbb{C})^{2}$ such that $\varphi^{*} \tilde{\sigma} \not \equiv 0$ and $\Delta \subseteq \operatorname{Supp}(\tilde{\sigma})$. Then $\Sigma=\operatorname{Supp} \Psi^{*}\left(\tilde{\sigma}_{0}\right)$ has the desired properties. By Theorems 2.2 and 2.7, we have a contradiction. Thus we see $\Phi \circ f \equiv \Phi \circ f_{0}$. By the assumption, we have $R_{j} \neq \emptyset$ for some $j$. Take a point $p \in R_{j}$. Then there exists an open neighborhood $U$ of $p$ such that $\left.\Phi\right|_{U}: U \rightarrow \Phi(U)$ is biholomorphic. Set $U^{\prime}=f_{0}^{-1}(U)$. It follows from $\Phi \circ f=\Phi \circ f_{0}$ and $f=f_{0}$ on $S$ that $f=f_{0}$ on $U^{\prime}$. Thus we see $f \equiv f_{0}$ by uniqueness of analytic continuation.

We next consider the case $\operatorname{dim} M=1$. Assume that $M$ is a compact Riemann surface with genus $g_{0}$. In the case $g_{0}=0$, we have the following unicity theorem for meromorphic functions on $X$ (cf. [1, Theorem 3.3]), which is closely related to the uniqueness problem of algebroid functions.

TheOREM 3.2. Let $f_{1}, f_{2}: X \rightarrow \mathbb{P}_{1}(\mathbb{C})$ be nonconstant holomorphic mappings. Let $a_{1}, \ldots, a_{d}$ be distinct points in $\mathbb{P}_{1}(\mathbb{C})$.

(1) Suppose that $\operatorname{Supp} f_{1}^{*} a_{j}=\operatorname{Supp} f_{2}^{*} a_{j}$ for all $j$. If $d \geq 2 s_{0}+3$, then $f_{1}$ and $f_{2}$ are identical on $X$.

(2) Suppose that $\operatorname{Supp}_{1} f_{1}^{*} a_{j}=\operatorname{Supp}_{1} f_{2}^{*} a_{j}$ for all $j$. If $d \geq 4 s_{0}+3$, then $f_{1}$ and $f_{2}$ are identical on $X$.

Proof. Let $M=\mathbb{P}_{1}(\mathbb{C})$ in Theorem 3.1. Assume that $k_{j}=+\infty$ for all $j$. Then we see $L_{0} \otimes K_{\mathbb{P}_{1}(\mathbb{C})}=\left(d-\left(2 s_{0}+2\right)\right) H$. Hence $L_{0} \otimes K_{\mathbb{P}_{1}(\mathbb{C})}$ is ample provided that $d \geq 2 s_{0}+3$. Thus Theorem 3.1 yields that $f_{1}$ and $f_{2}$ are identical. Next, let $k_{j}=1$ for all $j$. Note that at least one $\operatorname{Supp}_{1} f_{1}^{*} a_{j}$ is not empty by Remark 1.11. In this case, we have $L_{0} \otimes K_{\mathbb{P}_{1}(\mathbb{C})}=\left(d-\left(4 s_{0}+2\right)\right) H$. Thus we have our conclusion.

We note that Theorem 3.2 is sharp. The following example due to H. Ueda (cf. [22]): 
EXAMPLE 3.3. We consider the integral

$$
z=\varphi(w):=\int_{0}^{w} \frac{1}{\sqrt{1-t^{4}}} d t
$$

on the unit disc in $\mathbb{C}$. Set $z_{1}=\varphi(1), z_{2}=\varphi(\sqrt{-1}), z_{3}=\varphi(-1)$ and $z_{4}=\varphi(-\sqrt{-1})$. Then $\varphi$ maps the unit disc onto the square $z_{1} z_{2} z_{3} z_{4}$. By Schwarz's reflection principle, the inverse function of $z=\varphi(w)$ can be analytically continued over the complex plane $\mathbb{C}$, and the resulting function $w=f(z)$ is doubly periodic. Let $a_{1}=1, a_{2}=\sqrt{-1}, a_{3}=-1, a_{4}=-\sqrt{-1}$, $a_{5}=0$ and $a_{6}=\infty$. Set $f_{1}=f$ and $f_{2}=\sqrt{-1} f$. Then $\operatorname{Supp}_{1} f_{1}{ }^{*} a_{j}=$ $\operatorname{Supp}_{1} f_{2}{ }^{*} a_{j}$ for all $j$, but $f_{1} \not \equiv f_{2}$.

The uniqueness problem of holomorphic mappings into a compact Riemann surface with positive genus is not well studied (cf. [1], [4], [5] and [14]). In the case of $g_{0}=1$, we will discuss the uniqueness for holomorphic mappings into smooth elliptic curves and give some results in Section 5 . Assume that $g_{0} \geq 2$. Then there can be only restricted cases where there really exists a nonconstant holomorphic mapping $f: X \rightarrow M$. Now, we will prove the following unicity theorem, which gives an improvement of Theorem 3.6 in [1].

THEOREM 3.4. Let $f_{1}, f_{2}: X \rightarrow M$ be nonconstant holomorphic mappings. Let $a_{1}, \ldots, a_{d}$ be distinct points in $M$.

(1) Suppose that $\operatorname{Supp} f_{1}^{*} a_{j}=\operatorname{Supp} f_{2}^{*} a_{j}$ for all $j$. If $d>\max \left\{4 g_{0}\right.$, $\left.2\left(g_{0}+1\right)\left(s_{0}-1\right)\right\}$, then $f_{1}$ and $f_{2}$ are identical on $X$.

(2) Suppose that $\operatorname{Supp}_{1} f_{1}^{*} a_{j}=\operatorname{Supp}_{1} f_{2}^{*} a_{j}$ for all $j$. If $d>\max \left\{4 g_{0}\right.$, $\left.2\left(g_{0}+1\right)\left(2 s_{0}+1\right)-8 g_{0}\right\}$, then $f_{1}$ and $f_{2}$ are identical on $X$.

Proof. Since $H^{2}(M, \mathbb{Z}) \cong \mathbb{Z}$, we identify the Chern class $c_{1}(L)$ of $L$ with an integer. In general, $a_{i}$ and $a_{j}$ with $i \neq j$ are not linearly equivalent, but $c_{1}\left(\left[a_{i}\right]\right)=c_{1}\left(\left[a_{j}\right]\right)=1$. In the proof below, we only use this fact. Hence, without loss of generality, we may assume that all $a_{j}$ are lineary equivalent. Now, by Riemann-Roch's theorem, we have $\mu_{0} \leq g_{0}+1$ (see the proof of Theorem 2 in [12]). Take a meromorphic function $\Phi$ on $M$ such that $\operatorname{deg} \Phi=\mu_{0}$ and let $k_{j}=+\infty$ for all $j$. Then

$$
c_{1}\left(L_{0} \otimes K_{M}\right)=\left(d-2 \mu_{0}\left(s_{0}-1\right)-2 \mu_{0}\right)+\left(2 g_{0}-2\right) .
$$


Hence $L_{0} \otimes K_{M}$ is ample if $d>2 \mu_{0} s_{0}-\left(2 g_{0}-2\right)$. Thus, if $d>2\left(g_{0}+1\right)\left(s_{0}-1\right)$, then $\Phi \circ f_{1}=\Phi \circ f_{2}$. On the other hand, if $t$ is the number of branch points of $\Phi: X \rightarrow \mathbb{P}_{1}(\mathbb{C})$, then Riemann-Hurwitz' formula gives

$$
t \leq 2\left(g_{0}+\mu_{0}-1\right) \leq 4 g_{0}
$$

By Theorem 1.7, we have the following defect relation:

$$
\sum_{j=1}^{d} \Theta_{f_{1}}\left(a_{j}\right) \leq 4+2\left(g_{0}+1\right)\left(s_{0}-2\right) .
$$

Note that $4+2\left(g_{0}+1\right)\left(s_{0}-2\right)<2\left(g_{0}+1\right)\left(s_{0}-1\right)$. Thus, if the assumption of (1) is satisfied, then there exists at least one of the point $a_{j}$ such that Supp $f_{1}^{*} a_{j}$ is not empty and is not a branch point of $\Phi$. Therefore, as in the proof of Theorem 3.1, we have (1). Next, we assume that $k_{j}=1$ for all $j$. By Remark 1.11, at least one $\operatorname{Supp}_{1} f_{1}^{*} a_{j}$ is not empty. In this case

$$
c_{1}\left(2\left(L_{0} \otimes K_{M}\right)\right)=\left(d-4 \mu_{0}\left(s_{0}-1\right)-2 \mu_{0}\right)+\left(4 g_{0}-4\right) .
$$

Hence $L_{0} \otimes K_{M}$ is ample if $d>4 \mu_{0} s_{0}+2 \mu_{0}-4\left(g_{0}-1\right)$. By the above argument, we have our assertion.

Note that there have been examples of holomorphic mappings into compact Riemann surfaces that satisfy our condition (cf. [12]). The details on the uniqueness problem of holomorphic mappings into compact Riemann surfaces will be published elsewhere.

\section{§4. Meromorphic mappings into complex projective spaces}

In this section, we consider the case where $M=\mathbb{P}_{n}(\mathbb{C})$. In the remainder of this paper, we always assume that $l=2$. Note that, if $f: X \rightarrow \mathbb{P}_{n}(\mathbb{C})$ separates the fibers of $\pi: X \rightarrow \mathbb{C}^{m}$, we can always take $\mu_{0}=1$. Let $H$ be the hyperplane bundle over $\mathbb{P}_{n}(\mathbb{C})$. Then $\operatorname{Pic}\left(\mathbb{P}_{n}(\mathbb{C})\right) \cong \mathbb{Z}$ and $H$ is the generator of $\operatorname{Pic}\left(\mathbb{P}_{n}(\mathbb{C})\right)$ with $c_{1}(H)=1$. Let $K_{\mathbb{P}_{n}(\mathbb{C})}$ be the canonical bundle of $\mathbb{P}_{n}(\mathbb{C})$. It is well-known that

$$
K_{\mathbb{P}_{n}(\mathbb{C})}=-(n+1) H
$$

In this case, $F \in \operatorname{Pic}\left(\mathbb{P}_{n}(\mathbb{C})\right) \otimes \mathbb{Q}$ is big if and only if $F$ is ample. We also note that

$$
\operatorname{Pic}\left(\mathbb{P}_{n}(\mathbb{C})^{2}\right)=\pi_{1}^{*} \operatorname{Pic}\left(\mathbb{P}_{n}(\mathbb{C})\right) \oplus \pi_{2}^{*} \operatorname{Pic}\left(\mathbb{P}_{n}(\mathbb{C})\right)
$$


Hence we may assume that $\widetilde{L}=\widetilde{F}$. Since $\operatorname{Pic}\left(\mathbb{P}_{n}(\mathbb{C})\right) \cong \mathbb{Z}$, there exists a positive integer $d$ such that $L=d H$. There also exist positive integers $d_{j}$ such that $F_{j}=d_{j} H$ for $j=1,2$. Thus a holomorphic section of $\widetilde{L} \rightarrow \mathbb{P}_{n}(\mathbb{C})^{2}$ is a homogeneous polynomial $P(\xi ; \zeta)$ of degree $d_{1}$ in $\xi=\left(\xi_{0}, \ldots, \xi_{n}\right)$ and degree $d_{2}$ in $\zeta=\left(\zeta_{0}, \ldots, \zeta_{n}\right)$. Set $d_{0}=\max \left\{d_{1}, d_{2}\right\}$. Let $D$ be an effective divisor in $\mathbb{P}_{n}(\mathbb{C})$ with simple normal crossings. Assume that $D=D_{1}+\cdots+$ $D_{q}$ for some $D_{j} \in|L|$. Let $S$ be a hypersurface in $X$. We will show some theorems on the propagation of dependence, which are the prototype of our study in this paper. By Theorem 2.2, we now have the following theorem:

TheOREM 4.1. Let $D, S$ and $P(\xi ; \zeta)$ be as above. Let $f, g: X \rightarrow \mathbb{P}_{n}(\mathbb{C})$ be dominant meromorphic mappings such that $\operatorname{Supp}_{k} f^{*} D_{j}=\operatorname{Supp}_{k} g^{*} D_{j}=$ $S_{j}$ for some $k$ with $1 \leq k \leq+\infty$. Suppose that $P(f ; g)=0$ on $S$. If

$$
d\left(q-2\left(1+k^{-1}\right)\left(s_{0}-1\right)\right)>2 d_{0}+\left(1+k^{-1}\right)(n+1)
$$

then $P(f ; g)=0$ on $X$.

Proof. Let $M=\mathbb{P}_{n}(\mathbb{C})$ and $L=d H$ in Theorem 2.2. Since $K_{\mathbb{P}_{n}(\mathbb{C})}=$ $-(n+1) H$, we have

$$
L_{0} \otimes K_{M}=\left(\frac{k\left(d q-2 d_{0}\right)}{k+1}-2\left(s_{0}-1\right) d-n-1\right) H .
$$

Hence $L_{0} \otimes K_{M}$ is ample provided that the assumption in the theorem is satisfied. Therefore we have the desired conclusion.

In [5], S. J. Drouilhet dealt with the case of $n=1$ and obtained some theorems on the dependence of meromorphic functions. Related to this, we have the following:

TheOREM 4.2. Let $D$ and $D^{\prime}$ be hypersurfaces of degree $d$ which have only simple normal crossings. Let $f, g: \mathbb{C}^{m} \rightarrow \mathbb{P}_{n}(\mathbb{C})$ be dominant meromorphic mappings such that $\operatorname{Supp}_{k} f^{*} D=\operatorname{Supp}_{k} g^{*} D^{\prime}=Z$ as point sets $(1 \leq k \leq+\infty)$. Let $P(\xi ; \zeta)$ be as in Theorem 4.1. Suppose that $P(f ; g)=0$ on $Z$. If

$$
d \geq d_{1}+d_{2}+1+\left(1+k^{-1}\right)(n+1)
$$

then $P(f ; g)=0$ on $\mathbb{C}^{m}$. 
Proof. In this case, we have that

$$
n_{j}=\frac{d-n-1}{d} \quad \text { and } \quad p_{j}=\frac{k}{k+1}-\frac{n+1}{d}
$$

for $j=1,2$ in Proposition 2.16. We also see $\left[F_{j} / L\right]=d_{j} / d$. We can easily see that $n_{1}$ and $n_{2}$ are positive under the condition on $d$. Now, we have

$$
\begin{aligned}
Q:= & p_{1}-\frac{2 k}{k+1}\left[F_{1} / L\right]+n_{1}\left(p_{2}-\frac{2 k}{k+1}\left[F_{2} / L\right]\right) \\
= & \frac{k}{k+1}-\frac{n+1}{d}-\frac{2 k d_{1}}{(k+1) d} \\
& \quad+\frac{d-n-1}{d}\left(\frac{k}{k+1}-\frac{n+1}{d}-\frac{2 k d_{2}}{(k+1) d}\right) .
\end{aligned}
$$

For brevity, we set $u=k /(k+1)$. Then it is easy to see that $Q$ is positive provided that

$$
2 u d^{2}-2\left(u\left(d_{1}+d_{2}+1\right)+n+1\right) d+(n+1)^{2}>0 .
$$

If the assumption of Theorem 4.2 is satisfied, then the last inequality is valid, and hence $Q$ is positive. Therefore we have the desired conclusion by Proposition 2.16 .

If $X=\mathbb{C}, n=1$ and $k=+\infty$, then we have Drouilhet's theorem. He also gave some examples which show his result is sharp. In the case where $\left[L_{0}^{-1} \otimes K_{\mathbb{P}_{n}(\mathbb{C})}^{-1} / L\right]=0$, we have the following:

TheOREM 4.3. Let $f, g, D, S$ and $P(\xi ; \zeta)$ be as in Theorem 4.1. Let $k=1$ and $d=d_{0}+n+1$. Suppose that $P(f ; g)=0$ on $S$ and $q=4 s_{0}-2$. If $\delta_{f}(D)>0$, then $P(f ; g)=0$ on $X$.

Proof. In this case, $L_{0} \otimes K_{\mathbb{P}_{n}(\mathbb{C})}$ is trivial. Hence $\left[L_{0}^{-1} \otimes K_{\mathbb{P}_{n}(\mathbb{C})}^{-1} / L\right]=0$. Thus we have our assertion by Theorem 2.7 .

\section{§5. Holomorphic mappings into smooth elliptic curves}

We finally consider the case where $M$ is a smooth elliptic curve $E$. The uniqueness problem of holomorphic mappings into elliptic curves was first studied by E. M. Schmid [14] and he obtained the following unicity theorem: Let $f, g: R \rightarrow E$ be nonconstant holomorphic mappings, where $R$ is an open Riemann surface of a certain type. Then there exists a nonnegative integer 
$d$ depending only on $R$ such that, if $f^{-1}\left(a_{j}\right)=g^{-1}\left(a_{j}\right)$ for distinct $d+5$ points $a_{1}, \ldots, a_{d+5}$ in $E$, then $f$ and $g$ are identical. In the special case $R=\mathbb{C}$, we have $d=0$.

Until now, there have been only few studies on the uniqueness problem of holomorphic mappings $f: X \rightarrow E$ (cf. [4], [5] and [14]). In this section, we consider the problem to determine the condition which yields $f=\varphi(g)$ for an endomorphism $\varphi$ of the abelian group $E$. We first note the following fact: If $f: X \rightarrow E$ separates the fibers of $\pi: X \rightarrow \mathbb{C}^{m}$, then we can take $\mu_{0}=2$ (cf. [12, p. 286]). Let $L \in \operatorname{Pic}(E)$. Since $H^{2}(E, \mathbb{Z}) \cong \mathbb{Z}$, we identify the Chern class $c_{1}(L)$ of $L$ with an integer. We now consider the infimum $[F / L]$ of the set of rational numbers $\gamma$ such that $\gamma c_{1}(L)-c_{1}(F)$ is ample. We note that $[F / L]=\left[F / L^{\prime}\right]$ if $c_{1}(L)=c_{1}\left(L^{\prime}\right)$. Hence the conclusions of Theorem 2.15, Propositions 2.16 and 2.17 are still valid provided that $D_{j} \in$ $\left|q_{j} L_{j}\right|$ and all $c_{1}\left(L_{j}\right)$ are identical. We also note that $\tilde{\gamma}$ is not necessarily rational number in this section. It is well-known that

$$
\operatorname{Pic}\left(E^{2}\right) \neq \pi_{1}^{*} \operatorname{Pic}(E) \oplus \pi_{2}^{*} \operatorname{Pic}(E) .
$$

We denote by $[p]$ the point bundle determined by $p \in E$. Let $F_{1}=F_{2}=[p]$. Let $f, g: X \rightarrow E$ be nonconstant holomorphic mappings. We denote by $\operatorname{End}(E)$ the ring of endomorphisms of $E$. If $E$ has no complex multiplication, it is well-known that $\operatorname{End}(E) \cong \mathbb{Z}$. Hence $\varphi(x)=n x$ for some integer $n$.

We now seek conditions which yield $g=\varphi(f)$ for some $\varphi \in \operatorname{End}(E)$. To this end, we first remark that the number of the totally ramified value for nonconstant holomorphic mappings $f: X \rightarrow E$ is at most $8 s_{0}-8$. Let $\varphi \in \operatorname{End}(E)$ and consider a curve

$$
\widetilde{S}=\{(x, y) \in E \times E ; y=\varphi(x)\}
$$

in $E \times E$. Let $\widetilde{L}$ be the line bundle $[\widetilde{S}]$ determined by $\widetilde{S}$. In this section, $\tilde{\gamma}$ denotes the infimum of rational numbers such that $\gamma \widetilde{F} \otimes[\widetilde{S}]^{-1}$ is ample. Then we essentially use the following theorem proved by T. Katsura:

Theorem (Katsura). Let $\tilde{\gamma}$ be as above. Then $\tilde{\gamma}=\operatorname{deg} \varphi+1$.

By the above theorem, we have the following corollary (cf. [16, p. 89]):

Corollary. Let $n$ be an integer. If $\varphi \in \operatorname{End}(E)$ is an endomorphism defined by $\varphi(x)=n x$, then $\tilde{\gamma}=n^{2}+1$. 
If $E$ has no complex multiplication, we can give the value of $\tilde{\gamma}$ by direct calculation of Chern forms. This method is based on the existence of valence of correspondence and hence does not work in the case where $E$ has complex multiplication (cf. [7, p. 286]). We give the proof of Katsura's theorem in the next section.

TheOrem 5.1. Let $f, g$ and $\varphi$ be as above. Let $D_{1}=\left\{a_{1}, \ldots, a_{d}\right\}$ be a set of $d$ points and $\varphi$ a endomorphism of $E$. Set $D_{2}=\varphi\left(D_{1}\right)$. Assume that the number of points in $D_{2}$ is also d. Suppose that $\operatorname{Supp}_{k} f^{*} D_{1}=$ $\operatorname{Supp}_{k} g^{*} D_{2}$ for some $k$. If $d>2(\operatorname{deg} \varphi+1)+8\left(s_{0}-1\right)\left(1+k^{-1}\right)$, then $g=\varphi(f)$.

Proof. Note that $\left[D_{1}\right]$ may be not equal to $\left[D_{2}\right]$ but $c\left(\left[D_{1}\right]\right)=c\left(\left[D_{2}\right]\right)=$ $d$. We take a line bundle $L \rightarrow E$ with $c(L)=d$. In this case, we may assume that

$$
G_{0}=\left(\frac{k}{k+1}-8\left(s_{0}-1\right)\right) L \otimes\left(-\frac{2 \tilde{\gamma} k}{k+1}\right) F_{0} .
$$

Thus the assumption of Theorem 5.1 yields that $G_{0}$ is ample. We have now completed the proof of our theorem.

Note that, by Remark 1.11, $\operatorname{Supp}_{1} f^{*} D_{1}$ is not empty under the condition in Theorem 5.1. In the above theorem, we assume that the cardinality $\sharp D_{2}$ of the point set $D_{2}$ equals $d$. However, it may happen that $\sharp D_{2}<d$. For example, if $\varphi(x)=n x(n \in \mathbb{Z})$ and there exists at least one pair $(i, j)$ such that $a_{i}-a_{j}$ is $n$-torsion point, then $\sharp D_{2}<d$. In this case, by making use of Proposition 2.17, we have the following:

TheOrem 5.2. Let $f, g: \mathbb{C}^{m} \rightarrow E$ be nonconstant holomorphic mappings. Let $D_{1}=\left\{a_{1}, \ldots, a_{d}\right\}$ be a set of $d$ points and $\varphi \in \operatorname{End}(E)$. Set $D_{2}=\varphi\left(D_{1}\right)$. Assume that the number of points in $D_{2}$ is $d^{\prime}$. Suppose that $\operatorname{Supp}_{1} f^{*} D_{1}=\operatorname{Supp}_{1} g^{*} D_{2}$. If $d d^{\prime}>\left(d+d^{\prime}\right)(\operatorname{deg} \varphi+1)$, then $g=\varphi(f)$.

Proof. We take line bundles $L_{j}$ over $E$ such that $D_{j} \in\left|L_{j}\right|$. In Proposition 2.17 , let $n_{1}=n_{2}=1$ and $p_{1}=p_{2}=k /(k+1)$. We also have $\left[F_{1} / L_{1}\right]=1 / d$ and $\left[F_{2} / L_{2}\right]=1 / d^{\prime}$. Hence

$$
p_{1}-\frac{2 \tilde{\gamma} k}{k+1}\left[F_{1} / L_{1}\right]+n_{1} p_{2}-\frac{2 \tilde{\gamma} e_{0} k}{n_{2}(k+1)}\left[F_{2} / L_{2}\right]
$$

can be written as

$$
P:=\frac{k}{k+1}-\frac{2 \tilde{\gamma} k}{d(k+1)}+\frac{k}{k+1}-\frac{2 \tilde{\gamma} k}{d^{\prime}(k+1)} .
$$


If $d d^{\prime}>\left(d+d^{\prime}\right) \tilde{\gamma}$, then $P>0$. Hence the assumption of Proposition 2.17 is satisfied. This proves Theorem 5.2.

Corollary 5.3. Let $f$ and $g$ be as in Theorem 5.2. Let $D_{1}=\left\{a_{1}, \ldots\right.$, $\left.a_{d}\right\}$ be a set of $d$ points and set $D_{2}=\left\{n a_{1}, \ldots, n a_{d}\right\}$ for some integer $n$. Assume that the number of points in $D_{2}$ is $d^{\prime}$. Suppose that $\operatorname{Supp}_{1} f^{*} D_{1}=$ $\operatorname{Supp}_{1} g^{*} D_{2}$. If $d d^{\prime}>\left(d+d^{\prime}\right)\left(n^{2}+1\right)$, then $g=n f$.

In the case where $X=\mathbb{C}, \operatorname{Supp} f^{*} D_{1}=\operatorname{Supp} g^{*} D_{2}$ and $E$ has no complex multiplication, we have Drouilhet's theorem (cf. [5, Theorem 6]). We do not know whether Theorem 5.2 is sharp or not. However, if the condition $d d^{\prime}>\left(d+d^{\prime}\right)(\operatorname{deg} \varphi+1)$ is not satisfied, then it is not necessarily true that $g=\varphi(f)$.

EXAMPLE 5.4. Let $\varphi \in \operatorname{End}(E)$ be an endomorphism defined by $\varphi(x)=2 x$. Define $f, g: \mathbb{C} \rightarrow E$ by $f(z)=\bar{\pi}(x)$ and $g(z)=-2 \bar{\pi}(x)$, where $\bar{\pi}: \mathbb{C} \rightarrow E$ be the universal covering mapping. Let $D_{1}=\{x \in E ; 4 x=0\}$. Then $D_{2}=\varphi\left(D_{1}\right)=2 D_{1}$. It is clear that $\operatorname{Supp}_{1} f^{*} D_{1}=\operatorname{Supp}_{1} g^{*} D_{2}$. In this case, $d=16, d^{\prime}=4$ and $\operatorname{deg} \varphi+1=5$. Thus we have

$$
d d^{\prime}-\left(d+d^{\prime}\right)(\operatorname{deg} \varphi+1)=-36<0
$$

and $g \neq \varphi(f)$.

The following unicity theorem is a direct conclusion of Theorem 5.1:

Theorem 5.5. Let $a_{1}, \ldots, a_{d}$ be distinct points in E. Let $f, g: X \rightarrow E$ be nonconstant holomorphic mappings. Suppose that $\operatorname{Supp}_{k} f^{*} a_{j}=\operatorname{Supp}_{k}$ $g^{*} a_{j}$ for all $j$, where $1 \leq k \leq+\infty$. If $d>8 s_{0}-4+8 k^{-1}\left(s_{0}-1\right)$, then $f$ and $g$ are identical.

In the case of $X=\mathbb{C}^{m}$, we have the following:

Theorem 5.6. Let $a_{1}, \ldots, a_{d}$ be distinct points in E. Let $f, g: \mathbb{C}^{m} \rightarrow$ $E$ be nonconstant holomorphic mappings. Suppose that $\operatorname{Supp}_{1} f^{*} a_{j}=\operatorname{Supp}_{1}$ $g^{*} a_{j}$ for all $j$. If $d \geq 5$, then $f$ and $g$ are identical.

We give here the concluding remark. If we choose special points of $E$, we obtain an example which yields that Theorem 5.6 is sharp. Indeed, let $a_{1}, \ldots, a_{4}$ be two-torsion points in $E$ and let $\wp$ be the Weierstrass $\wp$ 
function. If $f_{1}^{*} a_{j}=f_{2}^{*} a_{j}$ for $j=1, \ldots, 4$, it is easy to see that $\wp \circ f_{1}=\wp \circ f_{2}$ by Nevanlinna's four points theorem (cf. [10, p. 122]). Hence $f_{1}=f_{2}$ or $f_{1}=-f_{2}$. Since $p \mapsto-p(p \in E)$ is an automorphism of $E$, it is acceptable that $f_{1}$ and $f_{2}$ are essentially identical. In this example, it seems that the structure of the function field of $E$ affects strongly the uniqueness problem for holomorphic mappings. We conjecture that, if we take "generic points" in $E$, then the number of points that implies the unicity of holomorphic mappings is less than five. Further studies in this direction are expected.

\section{§6. Appendix: Proof of Katsura's theorem}

In this section we give the proof of Katsura's theorem, which is the original proof due to T. Katsura.

Let $E$ be a smooth elliptic curve as in Section 5. Let $E_{1}=E_{2}=E$ and $\varphi \in \operatorname{End}(E)$. Then we regard $\varphi$ as an isogeny $\varphi: E_{1} \rightarrow E_{2}$. Set $A=E_{1} \times E_{2}$ and

$$
\widetilde{S}=\left\{(x, \varphi(x)) ; x \in E_{1}\right\} \subseteq A .
$$

Let $C$ be an irreducible curve in $A$. We denote by $\pi(C)$ the virtual genus of $C$, that is,

$$
\pi(C)=\frac{C^{2}+\left(C \cdot K_{A}\right)}{2}+1
$$

Since $K_{A}$ is trivial, we have

$$
\pi(C)=\frac{C^{2}+2}{2} .
$$

Since $\widetilde{S} \cong E_{1}$, it is clear that $\widetilde{S}$ is a smooth elliptic curve. Thus $\widetilde{S}^{2}=0$. Set $\widetilde{D}=E_{1} \times\{0\}+\{0\} \times E_{2}$. Then $\widetilde{D}^{2}=2$. For brevity, we write $E_{1}$ for $E_{1} \times\{0\}$ and $E_{2}$ for $\{0\} \times E_{2}$ in what follows. Then we set

$$
\tilde{\gamma}=\inf \{\gamma \in \mathbb{Q} ; \gamma \widetilde{D}-\widetilde{S} \text { is ample }\} .
$$

For the proof of the theorem, we need some lemmas. The following lemma directly follows from the definition.

Lemma 6.1. Let $\widetilde{S}, E_{1}$ and $E_{2}$ be as above. Then

$$
\left(\widetilde{S} \cdot E_{1}\right)=\operatorname{deg} \varphi \quad \text { and } \quad\left(\widetilde{S} \cdot E_{2}\right)=1 .
$$


Lemma 6.2. Let $A$ be an Abelian surface, and $C_{1}$ and $C_{2}$ irreducible curves in $A$. Denote by $T_{x}: A \rightarrow A$ the translation by $x \in A$. If $\left(C_{1} \cdot C_{2}\right)=$ 0 , then $C_{1}$ and $C_{2}$ are smooth elliptic curves and $T_{x}^{*} C_{1}=C_{2}$ for some $x \in A$.

Proof. Take $y \in C_{1}$ and $z \in C_{2}$ and set $x=y-z$. Then $z \in T_{x}^{*} C_{1} \cap C_{2}$. If $T_{x}^{*} C_{1} \neq C_{2}$, then $\left(C_{1} \cdot C_{2}\right)=\left(T_{x}^{*} C_{1} \cdot C_{2}\right)>0$. This contradicts the assumption. Thus we have $T_{x}^{*} C_{1}=C_{2}$ and hence

$$
C_{1}^{2}=\left(T_{x}^{*} C_{1} \cdot C_{1}\right)=\left(C_{2} \cdot C_{1}\right)=0 .
$$

Thus $\pi\left(C_{1}\right)=1$. This implies that $C_{1}$ is a smooth elliptic curve and so is $\mathrm{C}_{2}$.

Lemma 6.3. Let $A$ be an Abelian surface, and $C_{1}$ and $C_{2}$ irreducible curves in $A$. If $\left(C_{1} \cdot C_{2}\right)=1$, then $C_{1}$ and $C_{2}$ are smooth elliptic curves and $A \cong C_{1} \times C_{2}$.

Proof. Suppose that $C_{1}^{2}>0$. Set $\xi=\left(C_{1}^{2}\right) C_{2}-\left(C_{1} \cdot C_{2}\right) C_{1}$. Then we have $\left(C_{1} \cdot \xi\right)=0$. By Hodge's index theorem, we see $\xi^{2} \leq 0$. Then we get

$$
C_{1}^{2} \cdot C_{2}^{2} \leq\left(C_{1} \cdot C_{2}\right)^{2}=1 .
$$

It follows from $C_{2}^{2}=2 \chi\left(\mathcal{O}_{A}\left(C_{2}\right)\right)$ that $C_{2}^{2}$ is even. Hence $C_{2}^{2}=0$. This implies that $C_{2}$ is a smooth elliptic curve. Without loss of generality, we may assume that $C_{2}$ contains an identity element of $A$. Hence $C_{2}$ is an Abelian subvariety of $A$. Thus we have a fiber space

$$
\psi: A \rightarrow A / C_{2} .
$$

It follows from $\psi^{-1}(0)=C_{2}$ and $\left(C_{1} \cdot C_{2}\right)=1$ that $C_{1}$ is a section of $\psi$. Thus $C_{1} \cong A / C_{2}$ is a smooth elliptic curve, and hence $C_{1}^{2}=0$. This is absurd. Therefore $C_{1}^{2}=0$. This implies that $C_{1}$ is a smooth elliptic curve. By the same method, we have $C_{2}^{2}=0$ and $C_{2}$ is a smooth elliptic curve. We may assume that both of $C_{1}$ and $C_{2}$ are Abelian subvarieties. We define $\Phi: C_{1} \times C_{2} \rightarrow A$ by $\Phi(x, y)=x+y$. Since $\left(C_{1} \cdot C_{2}\right)=1$, we infer that $\Phi$ gives an isomorphism.

Lemma 6.4. (A. Weil) Let $A$ be an Abelian surface and $\Theta$ an effective divisor on $A$. Suppose that $\Theta^{2}=2$. Then one of the following occurs.

(1) $\Theta$ is a smooth curve with genus two.

(2) There exists smooth elliptic curves $C_{1}$ and $C_{2}$ with $\left(C_{1} \cdot C_{2}\right)=1$ such that $\Theta=C_{1}+C_{2}$. 
Proof. Let $\Theta=\sum_{i=1}^{l} n_{i} \Theta_{i}$, where $\Theta_{i}$ are distinct irreducible components of $\Theta$. Then

$$
\Theta^{2}=\sum_{i} n_{i}^{2} \Theta_{i}^{2}+\sum_{i<j} 2 n_{i} n_{j}\left(\Theta_{i} \cdot \Theta_{j}\right)=2
$$

If $l=1$, then $n_{1}=1$ and $\Theta=\Theta_{1}$. Hence the virtual genus $\pi(\Theta)$ equals 2. Suppose that $\Theta$ is a singular curve. Since $A$ contains no rational curve, $\Theta$ is a singular elliptic curve. Let $\psi: \widetilde{\Theta} \rightarrow \Theta$ be the desingularization of $\Theta$. Then $\widetilde{\Theta}$ is a smooth elliptic curve, and hence $\phi$ is a composite of a homomorphism and a translation. Thus $\Theta$ is smooth. This is absurd. Thus we conclude that $\Theta$ is a smooth curve with genus two. Next we assume that $l \geq 2$. Suppose that all $\left(\Theta_{i} \cdot \Theta_{j}\right)=0$. By Lemma 6.2, we see that all $\Theta_{i}^{2}=0$. This contradicts the assumption. Hence, for at least one pair of $i$ and $j,\left(\Theta_{i} \cdot \Theta_{j}\right)>0$. In this case, it is easy to see that $l=2$ and $n_{1}=n_{2}=1$. We also have $\left(\Theta_{1} \cdot \Theta_{2}\right)=1$.

Now we return the proof of the theorem of Katsura. Set $\tilde{\gamma}^{\prime}=\operatorname{deg} \varphi+1$ and let $F=\tilde{\gamma}^{\prime} \widetilde{D}-\widetilde{S}$. Then we have $F^{2}=0$. On the other hand, we see $\left(F \cdot E_{1}\right)=1$ and $\left(F \cdot E_{2}\right)=\operatorname{deg} \varphi$. Since $\left(F+E_{1}\right)^{2}=2$, we have

$$
\chi\left(\mathcal{O}_{A}\left(F+E_{1}\right)\right)=\frac{\left(F+E_{1}\right)^{2}}{2}=1>0 .
$$

Hence $h^{0}\left(\mathcal{O}_{A}\left(F+E_{1}\right)\right)>0$ or $h^{2}\left(\mathcal{O}_{A}\left(F+E_{1}\right)\right)>0$. Since $\left(\left(F+E_{1}\right) \cdot \widetilde{D}\right)=$ $\operatorname{deg} \varphi+2>0$, we get $h^{0}\left(\mathcal{O}_{A}\left(F+E_{1}\right)>0\right.$. Then there exists an effective divisor $D$ such that

$$
F+E_{1} \sim D \quad \text { and } \quad D^{2}=2
$$

Suppose that $D$ is irreducible. It follows from $F \sim D-E_{1}$ that

$$
0=F^{2}=D^{2}-2\left(D \cdot E_{1}\right)=2-2\left(D \cdot E_{1}\right)
$$

Thus $\left(D \cdot E_{1}\right)=1$, which contradicts Lemmas 6.3 and 6.4. Therefore there exist smooth elliptic curves $C_{1}$ and $C_{2}$ such that $\left(C_{1} \cdot C_{2}\right)=1$ and $D=C_{1}+C_{2}$. Since $F \sim D-E_{1}$, we have $\left(D \cdot E_{1}\right)=1$ as above. Hence $\left(C_{1} \cdot E_{1}\right)+\left(C_{2} \cdot E_{1}\right)=1$. Without loss of generality, we may assume that $\left(C_{1} \cdot E_{1}\right)=1$ and $\left(C_{2} \cdot E_{1}\right)=0$. By making use of Lemma 6.2 , there exists a point $x \in A$ such that $C_{2}=T_{x}^{*} C_{1}$. Hence

$$
F \sim D-E_{1}=C_{1}+T_{x}^{*} E_{1}-E_{1}
$$


Thus $F$ and $C_{1}$ are algebraically equivalent. Note that, for an arbitrary curve $C$ in $A$, we have $(F \cdot C)=\left(C_{1} \cdot C\right) \geq 0$. Let $\varepsilon$ be a sufficiently small positive rational number. Then we have

$$
\left(\tilde{\gamma}^{\prime}+\varepsilon\right) \widetilde{D}-\widetilde{S}=\varepsilon \widetilde{D}+F
$$

We also note that, for an arbitrary curve $C$ in $A,(\widetilde{D} \cdot C)>0$ and $(F \cdot C) \geq 0$. Hence, for an arbitrary curve $C$ in $A$,

$$
\left(\left\{\left(\tilde{\gamma}^{\prime}+\varepsilon\right) \widetilde{D}-\widetilde{S}\right\} \cdot C\right)>0 .
$$

This implies $\tilde{\gamma}^{\prime} \geq \tilde{\gamma}$. On the other hand, we have

$$
\left(\tilde{\gamma}^{\prime}-\varepsilon\right) \widetilde{D}-\widetilde{S}=-\varepsilon \widetilde{D}+F
$$

By Lemmas 6.1 and 6.3 , we see that $\left(\widetilde{D} \cdot C_{1}\right)>0$ and $\left(F \cdot C_{1}\right)=0$. Hence we get

$$
\left(\left\{\left(\tilde{\gamma}^{\prime}-\varepsilon\right) \widetilde{D}-\widetilde{S}\right\} \cdot C_{1}\right)<0 .
$$

This implies $\tilde{\gamma}^{\prime} \leq \tilde{\gamma}$. Therefore we have $\tilde{\gamma}^{\prime}=\tilde{\gamma}$. We have now completed the proof of Katsura's theorem.

\section{REFERENCES}

[1] Y. Aihara, A unicity theorem for meromorphic mappings into compactified locally symmetric spaces, Kodai Math. J., 14 (1991), 392-405.

[2] Y. Aihara, The uniqueness problem of meromorphic mappings with deficiencies, Tohoku Math. J., 51 (1999), 315-328.

[3] Y. Aihara, Unicity theorems for meromorphic mappings with deficiencies, Complex Variables, 42 (2000), 259-268.

[4] S. J. Drouilhet, A unicity theorem for meromorphic mappings between algebraic varieties, Trans. Amer. Math. Soc., 265 (1981), 349-358.

[5] S. J. Drouilhet, Criteria for algebraic dependence of meromorphic mappings into algebraic varieties, Illinois J. Math., 26 (1982), 492-502.

[6] H. Fujimoto, Uniqueness Problem with truncated multiplicities in value distribution theory I, II, Nagoya Math. J., 152 (1998), 131-152; ibid. 155 (1999), 161-188.

[7] P. Griffiths and J. Harris, Principles of Algebraic Geometry, Wiley, New York, 1979.

[8] S. Ji, Uniqueness theorems without multiplicities in value distribution theory, Pacific J. Math., 135 (1988), 323-348.

[9] S. Kobayashi and T. Ochiai, Mappings into compact complex manifolds with negative first Chern class, J. Math. Soc. Japan, 23 (1971), 137-148.

[10] R. Nevanlinna, Le Théorème de Picard-Borel et la Théorie des Fonction Méromorphes, Gauthier-Villars, Paris, 1929. 
[11] J. Noguchi, Meromorphic mappings of covering spaces over $\mathbf{C}^{m}$ into a projective variety and defect relations, Hiroshima Math. J., 6 (1976), 265-280.

[12] J. Noguchi, Holomorphic mappings into closed Riemann surfaces, Hiroshima Math. J., 6 (1976), 281-290.

[13] L. Sario, General value distribution theory, Nagoya Math. J., 23 (1963), 213-229.

[14] E. M. Schmid, Some theorems on value distribution of meromorphic functions, Math. Z., 23 (1971), 561-580.

[15] H. Selberg, Algebroid Funktionen und Umkerfunktionen Abelscher Integrale, Avh. Norske Vid. Acad. Oslo, 8 (1934), 1-72.

[16] J. Silverman, The Arithmetic of Elliptic Curves, Springer-Verlag, Berlin-HeiderbergNew York, 1991.

[17] L. Smiley, Dependence theorems for meromorphic maps, Ph.D. Thesis, Notre Dame Univ. (1979).

[18] L. Smiley, Geometric conditions for the unicity of holomorphic curves, Contemporary Math., 25 (1983), 149-154.

[19] W. Stoll, The Ahlfors-Weyl theory of meromorphic maps on parabolic manifolds, Proc. Value Distribution Theory, Joensuu 1981 (I. Laine et al., eds.), Lect. Notes in Math. 981, Springer-Verlag, Berlin-Heiderberg-New York (1983), pp. 101-219.

[20] W. Stoll, Algebroid reduction of Nevanlinna theory, Complex Analysis III, Proc. 1985-1986 (C. A. Berenstein, ed.), Lect. Notes in Math. 1277, Springer-Verlag, Berlin-Heiderberg-New York (1987), pp. 131-241.

[21] W. Stoll, Propagation of dependences, Pacific J. Math., 139 (1989), 311-336.

[22] H. Ueda, Unicity theorems for meromorphic or entire functions, Kodai Math. J., 3 (1980), 212-223.

Division of Liberal Arts

Numazu College of Technology

3600 Ooka

Numazu

Shizuoka, 410-8501

Japan

aihara@la.numazu-ct.ac.jp 